\title{
Metal-Based Ionic Liquids in Oxidative Desulfurization: A Critical Review
}

Fen Liu, Jiang Yu*, Abdul Basit Qazi, Li Zhang, and Xueke Liu

Research Group of Environmental Catalysis and Separation Process, Beijing Key Laboratory of Energy Environmental Catalysis, College of

Chemical Engineering, Beijing University of Chemical Technology, Beijing 100029, China

Corresponding author: Jiang Yu-Research Group of Environmental Catalysis and Separation Process, Beijing Key Laboratory of Energy Environmental Catalysis, College of Chemical Engineering, Beijing University of Chemical Technology, Beijing 100029, China; Email: yujiang@mail.buct.edu.cn 


\section{Content}

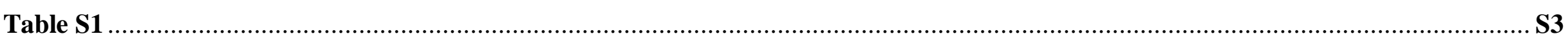

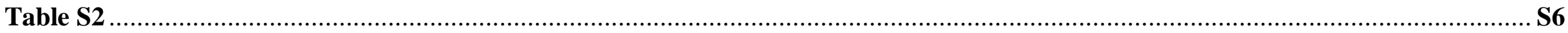

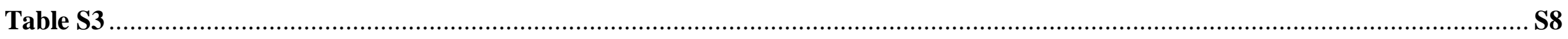

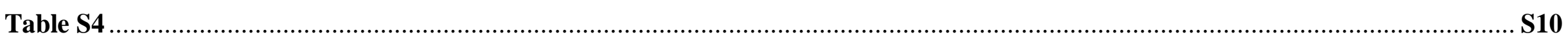

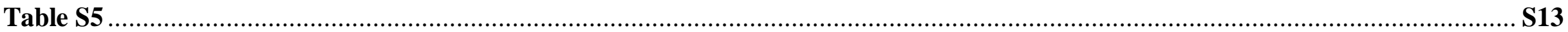

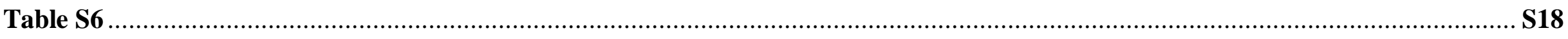

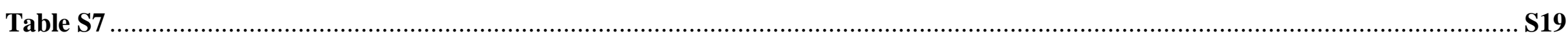

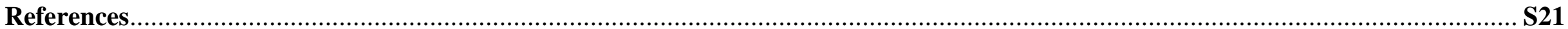


Table S1. Characterization of properties and structure of metal chloride ILs.

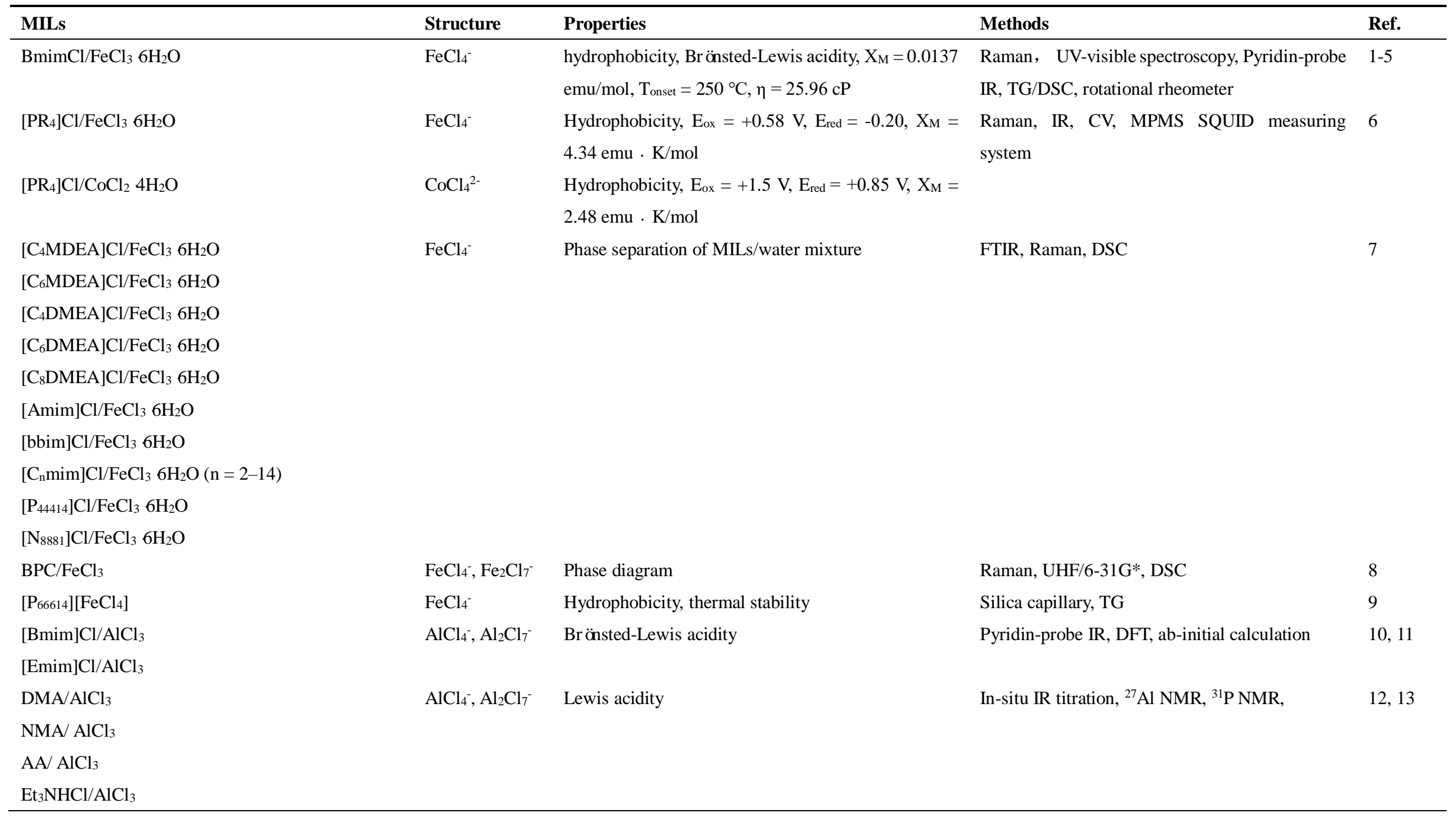


Table S1. Continued.

\begin{tabular}{|c|c|c|c|c|}
\hline $\mathrm{BmimCl} / \mathrm{FeCl}_{3}$ & $\begin{array}{l}\mathrm{FeCl}_{4}^{-}, \mathrm{Fe}_{2} \mathrm{Cl}_{7}^{-} \\
\text {nanostructure }\end{array}$ & Brönsted-Lewis acidity, $\gamma=46.03 \times 10^{-4} \mathrm{~N} / \mathrm{m}$ & $\begin{array}{l}\text { Raman, IR, FF-TEM, XPS, FT-IR, NMR, DFT, } \\
\text { ab-initial calculation, Westphal balance, least- } \\
\text { square method, Interstice model }\end{array}$ & $1,14-16$ \\
\hline$\left[\mathrm{C}_{4} \mathrm{mim}\right] \mathrm{Cl} / \mathrm{FeCl}_{3} \cdot 6 \mathrm{H}_{2} \mathrm{O}$ & \multirow[t]{8}{*}{$\mathrm{FeCl}_{4}^{-}$} & $\mathrm{M}_{\mathrm{r}} / \mathrm{Ms}=0.095$ & \multirow{8}{*}{$\begin{array}{l}\text { Raman, UV-visible spectrum, MPMS SQUID } \\
\text { measuring system }\end{array}$} & \multirow[t]{8}{*}{17} \\
\hline$\left[\mathrm{C}_{6} \operatorname{mim}\right] \mathrm{Cl} / \mathrm{FeCl}_{3} \cdot 6 \mathrm{H}_{2} \mathrm{O}$ & & $\mathrm{M}_{\mathrm{r}} / \mathrm{Ms}=0.160$ & & \\
\hline$\left[\mathrm{C}_{8} \mathrm{mim}\right] \mathrm{Cl} / \mathrm{FeCl}_{3} \cdot 6 \mathrm{H}_{2} \mathrm{O}$ & & $\mathrm{M}_{\mathrm{r}} / \mathrm{Ms}=0.095$ & & \\
\hline$\left[\mathrm{C}_{10 \mathrm{mim}}\right] \mathrm{Cl} / \mathrm{FeCl}_{3} \cdot 6 \mathrm{H}_{2} \mathrm{O}$ & & $\mathrm{M}_{\mathrm{r}} / \mathrm{Ms}=0.133$ & & \\
\hline$\left[\mathrm{C}_{12} \mathrm{mim}\right] \mathrm{Cl} / \mathrm{FeCl}_{3} \cdot 6 \mathrm{H}_{2} \mathrm{O}$ & & $\mathrm{M}_{\mathrm{r}} / \mathrm{Ms}=0.217$ & & \\
\hline$\left[\mathrm{C}_{14} \mathrm{mim}\right] \mathrm{Cl} / \mathrm{FeCl}_{3} \cdot 6 \mathrm{H}_{2} \mathrm{O}$ & & $\mathrm{M}_{\mathrm{r}} / \mathrm{Ms}=0.223$ & & \\
\hline \multirow[t]{2}{*}[\mathrm{C}_{16\mathrm{mim}}]{$\mathrm{Cl} / \mathrm{FeCl}_{3} \cdot 6 \mathrm{H}_{2} \mathrm{O}$} & & $\mathrm{M}_{\mathrm{r}} / \mathrm{Ms}=0.186$ & & \\
\hline & & $\mathrm{T}_{\text {onset }}=250-310^{\circ} \mathrm{C}$ & & \\
\hline$\left[\mathrm{C}_{4}(\mathrm{mim})_{2}\right] \mathrm{Cl}_{2} / \mathrm{FeCl}_{3}$ & $\mathrm{FeCl}_{4}^{-}, \mathrm{Fe}_{2} \mathrm{Cl}_{7}^{-}$ & $\mathrm{T}_{\text {onset }}=340^{\circ} \mathrm{C}, \mathrm{X}_{\mathrm{g}}=4.39 \times 10^{-5} \mathrm{emu} / \mathrm{g}$ & ESI-MS, FTIR, UV-vis, TG-DSC & 18 \\
\hline$[\mathrm{Omim}] \mathrm{Cl} / \mathrm{FeCl}_{3}$ & $\mathrm{FeCl}_{4}^{-}$ & $\mathrm{T}_{\text {onset }}=370^{\circ} \mathrm{C}$ & FTIR, ${ }^{1} \mathrm{H}$ NMR, TG-DSC & 19 \\
\hline$[\mathrm{Emim}]\left[\mathrm{FeCl}_{4}\right]$ & $\mathrm{FeCl}_{4}^{-}$ & $\mathrm{T}_{\text {onset }}=280^{\circ} \mathrm{C}, \eta=14 \mathrm{cP}, \sigma=1.8 \times 10^{-2} \mathrm{~S} / \mathrm{cm}$ & DSC, least-square method & 20 \\
\hline$[\mathrm{Emim}]\left[\mathrm{Fe}_{0.5} \mathrm{Ga}_{0.5} \mathrm{Cl}_{4}\right]$ & {$\left[\mathrm{Fe}_{0.5} \mathrm{Ga}_{0.5} \mathrm{Cl}_{4}\right]^{-}$} & $\mathrm{T}_{\text {onset }}=190^{\circ} \mathrm{C}, \eta=12 \mathrm{cP}, \sigma=2.0 \times 10^{-2} \mathrm{~S} / \mathrm{cm}$ & & \\
\hline$\left[\left(\mathrm{C}_{4} \mathrm{H}_{9}\right)_{2}-\mathrm{bim}\right] \mathrm{Cl} / \mathrm{FeCl}_{3}$ & $\mathrm{FeCl}_{4}^{-}, \mathrm{Fe}_{2} \mathrm{Cl}_{7}^{-}$ & $\begin{array}{l}\mathrm{T}_{\text {onset }}=265^{\circ} \mathrm{C}, \mathrm{E}_{\mathrm{ox}}=-0.687 \mathrm{~V}, \mathrm{E}_{\text {red }}=-0.775 \mathrm{~V}, \mathrm{X}_{\mathrm{M}}= \\
2.28 \mathrm{emu} \cdot \mathrm{K} / \mathrm{mol}\end{array}$ & $\begin{array}{l}\text { TG/DTG, DSC, CV, MPMS SQUID measuring } \\
\text { system }\end{array}$ & 21 \\
\hline $\mathrm{Et}_{3} \mathrm{NHCl} / \mathrm{FeCl}_{3}$ & $\mathrm{FeCl}_{4}^{-}$ & $\mathrm{T}_{\text {onset }}=250^{\circ} \mathrm{C}$ & TG-DSC & 22 \\
\hline$\left[\mathrm{P}_{66614}\right]\left[\mathrm{FeCl}_{4}\right]$ & $\mathrm{FeCl}_{4}^{-}$ & Viscosity varied with magnetic field & ${ }^{1} \mathrm{H}$ NMR, capillary viscometry & 23 \\
\hline 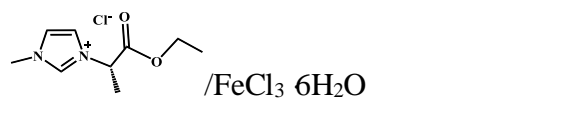 & $\mathrm{FeCl}_{4}^{-}$ & $\mathrm{T}_{\text {onset }}=264.94{ }^{\circ} \mathrm{C}, \mathrm{X}_{\mathrm{M}}=0.01398 \mathrm{emu} / \mathrm{mol}$ & $\begin{array}{l}\text { Raman, FTIR, NMR, MPMS SQUID measuring } \\
\text { system }\end{array}$ & 24 \\
\hline$\left[\left(\mathrm{CH}_{3}\right)_{2} \mathrm{NR}^{1} \mathrm{R}^{2}\right] \mathrm{Cl} / \mathrm{FeCl}_{3}$ & $\mathrm{FeCl}_{4}^{-}$ & $\mathrm{T}_{\text {onset }}=250-310^{\circ} \mathrm{C}$ & Raman, DSC, TG & 25 \\
\hline \multicolumn{5}{|l|}{$\left(\mathrm{R}^{1}=\mathrm{CH}_{3}, \mathrm{C}_{4} \mathrm{H}_{9},\left(\mathrm{CH}_{2}\right)_{3} \mathrm{CN}\right.$} \\
\hline \multicolumn{5}{|l|}{$\left.\mathrm{R}^{2}=\mathrm{C}_{10} \mathrm{H}_{21}, \mathrm{C}_{16} \mathrm{H}_{33}, \mathrm{CH}_{2} \mathrm{CH}_{2} \mathrm{OH}, \mathrm{CH}_{2} \mathrm{C}_{6} \mathrm{H}_{5}\right)$} \\
\hline$[\mathrm{DHMTA}] \mathrm{Cl} / \mathrm{FeCl}_{3} \cdot 6 \mathrm{H}_{2} \mathrm{O}$ & $\mathrm{FeCl}_{4}^{-}$ & Self-assembly, magnetism & ${ }^{1} \mathrm{H}$ NMR, ${ }^{13} \mathrm{C}$ NMR, Raman, SQUID method & 26 \\
\hline$[\mathrm{PDHMTA}] \mathrm{Cl} / \mathrm{FeCl}_{3} \cdot 6 \mathrm{H}_{2} \mathrm{O}$ & & & & \\
\hline
\end{tabular}


Table S1. Continued.

\begin{tabular}{|c|c|c|c|c|}
\hline$[\mathrm{DTA}] \mathrm{Br} / \mathrm{FeCl}_{3}$ & $\mathrm{FeCl}_{3} \mathrm{Br}^{-}$ & $\mathrm{CMC}=13.6 \mathrm{mM}$ & ESI-MS, TGA, conductivity meter, MPMS & 27 \\
\hline$[\mathrm{TTA}] \mathrm{Br} / \mathrm{FeCl}_{3}$ & & $\mathrm{CMC}=1.90 \mathrm{mM}$ & SQUID system & \\
\hline$[\mathrm{CTA}] \mathrm{Br} / \mathrm{FeCl}_{3}$ & & $\mathrm{CMC}=0.82 \mathrm{mM}$ & & \\
\hline \multirow[t]{2}{*}[\mathrm{STA}]{$\mathrm{Br} / \mathrm{FeCl}_{3}$} & & $\mathrm{CMC}=0.22 \mathrm{mM}$ & & \\
\hline & & $\mathrm{T}_{\mathrm{d}}=212-231{ }^{\circ} \mathrm{C}$, magnetism & & \\
\hline$[\mathrm{Hnmp}] \mathrm{Cl} / \mathrm{FeCl}_{3}$ & $\mathrm{FeCl}_{4}^{-}$ & $\mathrm{T}_{\text {onset }}=140^{\circ} \mathrm{C}$ & FTIR, Raman, TG & 28 \\
\hline$[\mathrm{Bmim}] \mathrm{Cl} / \mathrm{MCl}_{2}$ & {$\left[\mathrm{MCl}_{4}\right]^{2-}$} & Conductivity, $\mathrm{T}_{\mathrm{d}}>300{ }^{\circ} \mathrm{C}$ & ${ }^{1} \mathrm{H}$ NMR, ${ }^{13} \mathrm{C}$ NMR, XRD, TGA & 29 \\
\hline \multicolumn{5}{|c|}{$(\mathrm{M}=\mathrm{Sn}, \mathrm{Cu}, \mathrm{Ni}, \mathrm{Mn}, \mathrm{Fe}, \mathrm{Co}, \mathrm{Zn}, \mathrm{Pt})$} \\
\hline$[\mathrm{Bmim}] \mathrm{Cl} / \mathrm{CuCl}$ & {$\left[\mathrm{CuCl}_{3}\right]^{2-}$} & $\begin{array}{l}\text { Glass transition and freezing temperature was } 267 \mathrm{~K} \\
\text { and } 324 \mathrm{~K} \text {, respectively }\end{array}$ & XRPD, DSC & 30 \\
\hline$[\mathrm{Hnoc}] \mathrm{Cl} / \mathrm{ZnCl}_{2}$ & $\begin{array}{l}\mathrm{ZnCl}_{3}^{-}, \mathrm{Zn}_{2} \mathrm{Cl}_{5}^{-}, \\
\mathrm{Zn}_{3} \mathrm{Cl}_{7}^{-}\end{array}$ & $\mathrm{T}_{\text {onset }}=100-200^{\circ} \mathrm{C}$ & ${ }^{1} \mathrm{H}$ NMR, negative ion MS, FTIR, TG & 31 \\
\hline$[\mathrm{HDBN}] \mathrm{Cl} / \mathrm{ZnCl}_{2}$ & $\mathrm{ZnCl}_{3}^{-}, \mathrm{Zn}_{2} \mathrm{Cl}_{5}^{-}$ & Lewis acidity, $\mathrm{T}_{\text {onset }}=420^{\circ} \mathrm{C}$ & ${ }^{1} \mathrm{H}$ NMR, negative ion MS, FTIR, TG/DTG & 32 \\
\hline$[\mathrm{ODBU}] \mathrm{Cl} / \mathrm{ZnCl}_{2}$ & $\mathrm{ZnCl}_{3}^{-}, \mathrm{Zn}_{2} \mathrm{Cl}_{5}^{-}$ & $\mathrm{T}_{\text {onset }}=415^{\circ} \mathrm{C}$ & ${ }^{1} \mathrm{H}$ NMR, negative ion MS, FTIR, TG/DTG & 33 \\
\hline
\end{tabular}


Table S2. Characterization of properties and structure of metal oxide ILs.

\begin{tabular}{|c|c|c|c|c|}
\hline MILs & Structure & Properties & Methods & Ref. \\
\hline$\left[\mathrm{C}_{4} \mathrm{VIM}\right]\left[\mathrm{PMoV}_{2}\right]$ & {$\left[\mathrm{PMoV}_{2}\right]^{-}$} & $\begin{array}{l}\text { Oxygen reduction at }-0.45 \mathrm{~V} \text {, and metal } \\
\text { reduction at }-0.95 \mathrm{~V}\end{array}$ & FTIR, XRD, SEM, XPS, CV & 34 \\
\hline$[\mathrm{Bmim}]_{3} \mathrm{PMo}_{12} \mathrm{O}_{40}$ & $\mathrm{PMo}_{12} \mathrm{O}_{40}{ }^{3-}$ & $\mathrm{T}_{\text {onset }}=300^{\circ} \mathrm{C}$, dissolving capacity of ILs & FTIR, TG/DSC, ${ }^{35} \mathrm{Cl}$ NMR & 35 \\
\hline$\left[\mathrm{C}_{16} \mathrm{mim}\right][\mathrm{PMoO}]$ & {$\left[\mathrm{PMoO}^{-}\right.$} & Hydrophobicity & FTIR, XRD, HRTEM, EDS & 36 \\
\hline $\begin{array}{l}(\mathrm{NKBu})_{7} \mathrm{PMo}_{12} \mathrm{O}_{42} \\
\left(\mathrm{NKC}_{12}\right)_{7} \mathrm{PMo}_{2} \mathrm{O}_{42}\end{array}$ & $\begin{array}{l}\text { Elemental analysis of } \mathrm{C}, \mathrm{H}, \mathrm{N}, \mathrm{Mo} \\
\text { and } \mathrm{P}\end{array}$ & Brönsted-Lewis acidity & $\begin{array}{l}{ }^{1} \mathrm{H} \text { NMR, }{ }^{13} \mathrm{C} \text { NMR, ESI-MS, IR, X- } \\
\text { ray fluorescence wave spectrometer }\end{array}$ & 37 \\
\hline$[\mathrm{PyPS}]_{3}\left(\mathrm{NH}_{4}\right)_{3} \mathrm{Mo}_{7} \mathrm{O}_{24}$ & $\left(\mathrm{NH}_{4}\right)_{3} \mathrm{Mo}_{7} \mathrm{O}_{24}{ }^{3-}$ & $\mathrm{T}_{\text {onset }}=250^{\circ} \mathrm{C}$ & XRD, FTIR, XPS, TG-DTA & 38 \\
\hline$[\mathrm{Bmim}]_{5}\left[\mathrm{PMo}{ }_{11} \mathrm{Co}\left(\mathrm{H}_{2} \mathrm{O}\right) \mathrm{O}_{39}\right]$ & {$\left[\mathrm{PMo}_{11} \mathrm{Co}\left(\mathrm{H}_{2} \mathrm{O}\right) \mathrm{O}_{39}\right]^{5-}$} & $\mathrm{T}_{\text {onset }}=310^{\circ} \mathrm{C}$ & $\begin{array}{l}\text { FTIR, UV-vis diffuse reflectance } \\
\text { spectra, TGA-DSC, }{ }^{31} \mathrm{P} \text { NMR }\end{array}$ & 39 \\
\hline$(\mathrm{TBA})_{5} \mathrm{~K}\left[\mathrm{a}_{1}-\mathrm{Hf}\left(\mathrm{H}_{2} \mathrm{O}\right)_{4} \mathrm{P}_{2} \mathrm{~W}_{17} \mathrm{O}_{61}\right]$ & {$\left[\mathrm{K}\left[\mathrm{a}_{1}-\mathrm{Hf}\left(\mathrm{H}_{2} \mathrm{O}\right)_{4} \mathrm{P}_{2} \mathrm{~W}_{17} \mathrm{O}_{61}\right]\right]^{5-}$} & Lewis acidity regulation & $\mathrm{IR},{ }^{31} \mathrm{P}$ NMR, ${ }^{183} \mathrm{~W}$ NMR, MS & 40 \\
\hline$(\mathrm{TBA})_{3.7} \mathrm{~K}_{0.3}\left[\mathrm{a}_{1}-\mathrm{Hf}\left(\mathrm{H}_{2} \mathrm{O}\right) \mathrm{PW}_{11} \mathrm{O}_{39}\right]$ & {$\left[\mathrm{K}_{0.3}\left[\mathrm{a}_{1}-\mathrm{Hf}\left(\mathrm{H}_{2} \mathrm{O}\right) \mathrm{PW}_{11} \mathrm{O}_{39}\right]\right]^{3.7-}$} & & & \\
\hline$\left[\left(\mathrm{C}_{8} \mathrm{H}_{17}\right)_{3} \mathrm{NCH}_{3}\right]_{2} \mathrm{~W}_{6} \mathrm{O}_{19}$ & $\mathrm{~W}_{6} \mathrm{O}_{19}{ }^{2-}$ & $\mathrm{T}_{\text {onset }}=300^{\circ} \mathrm{C}$ & FTIR, UV-vis, TG & 41 \\
\hline$\left[\left(\mathrm{C}_{18} \mathrm{H}_{37}\right) \mathrm{N}\left(\mathrm{CH}_{3}\right)_{3}\right]_{7}\left[\mathrm{PW}_{11} \mathrm{O}_{39}\right]$ & {$\left[\mathrm{PW}_{11} \mathrm{O}_{39}\right]^{7-}$} & $\mathrm{T}_{\text {onset }}=200^{\circ} \mathrm{C}$ & FTIR, UV-vis, TG & 42 \\
\hline$\left[\left(\mathrm{CH}_{3}\right) \mathrm{N}\left(\mathrm{n}-\mathrm{C}_{8} \mathrm{H}_{17}\right)_{3}\right]_{2} \mathrm{~W}_{2} \mathrm{O}_{11}$ & $\mathrm{~W}_{2} \mathrm{O}_{11}{ }^{2-}$ & $\mathrm{T}_{\text {onset }}=110^{\circ} \mathrm{C}$ & TG-DSC & 43 \\
\hline$\left[\mathrm{C}_{4} \mathrm{mim}_{3}\left[\mathrm{PMo}_{4} \mathrm{O}_{24}\right]\right.$ & {$\left[\mathrm{PMo}_{4} \mathrm{O}_{24}\right]^{3-}$} & $\mathrm{T}_{\text {onset }}=158^{\circ} \mathrm{C}$ & FTIR, TG-DSC & 44 \\
\hline $\begin{array}{l}{\left[\left(\mathrm{CH}_{3}\right)\left(\mathrm{C}_{18} \mathrm{H}_{37}\right) \mathrm{N}\left[\left(\mathrm{CH}_{2} \mathrm{CH}_{2} \mathrm{O}\right)_{n} \mathrm{H}\right]\left[\left(\mathrm{CH}_{2} \mathrm{CH}_{2} \mathrm{O}\right)_{m}\right.\right.} \\
\mathrm{H}]]_{3}\left[\mathrm{PW}_{12} \mathrm{O}_{40}\right]\end{array}$ & {$\left[\mathrm{PW}_{12} \mathrm{O}_{40}\right]^{3-}$} & $\mathrm{T}_{\text {onset }}=160^{\circ} \mathrm{C}$, high conductivity & XRD, TG-DSC & 45 \\
\hline$\left[\left(\mathrm{C}_{6} \mathrm{H}_{13}\right)_{3} \mathrm{P}\left(\mathrm{C}_{14} \mathrm{H}_{29}\right)\right]_{2}\left[\mathrm{~W}_{6} \mathrm{O}_{19}\right]$ & {$\left[\mathrm{W}_{6} \mathrm{O}_{19}\right]^{2-}$} & $\begin{array}{l}\mathrm{T}_{\mathrm{g}}=-48{ }^{\circ} \mathrm{C}, \mathrm{T}_{\text {onset }}=160{ }^{\circ} \mathrm{C}, \eta=1.1 \times 10^{5} \\
\mathrm{mPa} \cdot \mathrm{S}, \sigma=4.5 \times 10^{-4} \mathrm{mS} / \mathrm{cm}, \mathrm{E}_{1 / 2}=-1.25 \mathrm{~V}\end{array}$ & EXAFS, CV, SAXS, TG-DSC & 46 \\
\hline$[\mathrm{MIMPS}]_{7} \mathrm{SiW}_{9} \mathrm{~V}_{3} \mathrm{O}_{40}$ & {$\left[\mathrm{SiW}_{9} \mathrm{~V}_{3} \mathrm{O}_{40}\right]^{7-}$} & Gel state to liquid state at $87-91^{\circ} \mathrm{C}, 10^{-4}$ to $10^{-}$ & FTIR, UV-vis, XRD, CV, DTA & 47 \\
\hline [MIMPS] ${ }_{5} \mathrm{SiMo}_{11} \mathrm{VO}_{40}$ & {$\left[\mathrm{SiMo}_{11} \mathrm{VO}_{40}\right]^{5-}$} & ${ }^{3} \mathrm{~S} / \mathrm{cm}$, formal potentials ranged from $0.35 \mathrm{~V}$ & & \\
\hline$[\mathrm{MIMPS}]_{5} \mathrm{SiW}_{11} \mathrm{VO}_{40}$ & {$\left[\mathrm{SiW}_{11} \mathrm{VO}_{40}\right]^{5-}$} & to $0.22 \mathrm{~V}$, conductivity & & \\
\hline$(\mathrm{THTP})_{4}\left[\mathrm{PW}_{11} \mathrm{O}_{39} \mathrm{Fe}^{\mathrm{III}}\left(\mathrm{H}_{2} \mathrm{O}\right)\right]$ & {$\left[\mathrm{PW}_{11} \mathrm{O}_{39} \mathrm{Fe}^{\mathrm{III}}\left(\mathrm{H}_{2} \mathrm{O}\right)\right]^{4-}$} & $\begin{array}{l}\mathrm{T}_{\text {onset }}=300{ }^{\circ} \mathrm{C}, \mathrm{T}_{\mathrm{g}}=-24.6{ }^{\circ} \mathrm{C}, \mathrm{E}_{1 / 2}=-0.272 \mathrm{~V} \\
\text { for } \mathrm{Fe}^{\mathrm{III}} / \mathrm{Fe}^{\mathrm{II}}, \mathrm{E}_{1 / 2}=-0.522 \mathrm{~V} \text { and }-0.732 \mathrm{~V} \text { for } \\
\mathrm{W}^{\mathrm{III}} / \mathrm{W}^{\mathrm{II}}\end{array}$ & ${ }^{31} \mathrm{P}$ NMR, FTIR, TG-DSC, CV & 48 \\
\hline
\end{tabular}


Table S2. Continued.

$(\mathrm{Bmim})_{10}\left[\left(\mathrm{PW}_{11} \mathrm{O}_{39} \mathrm{Fe}^{\mathrm{III}}\right)_{2} \mathrm{O}\right] \cdot 0.5 \mathrm{H}_{2} \mathrm{O}$

$\left[\left(\mathrm{PW}_{11} \mathrm{O}_{39} \mathrm{Fe}^{\mathrm{III}}\right)_{2} \mathrm{O}\right]^{10-}$

Magnetism, $\mathrm{T}_{\mathrm{onset}}=300^{\circ} \mathrm{C}$

UV-vis,

$(\mathrm{Bmim})_{4}\left[\left(\mathrm{PW}_{11} \mathrm{O}_{39} \mathrm{Fe}^{\mathrm{III}}\left(\mathrm{H}_{2} \mathrm{O}\right)\right] \cdot \mathrm{H}_{2} \mathrm{O}\right.$

$\left[\left(\mathrm{PW}_{11} \mathrm{O}_{39} \mathrm{Fe}^{\mathrm{III}}\right]^{4-}\right.$

MALDI-MS

(Bmim) $)_{5} \mathrm{PMo}_{10} \mathrm{~V}_{2} \mathrm{O}_{40}$

$\left[\mathrm{PMo}_{10} \mathrm{~V}_{2} \mathrm{O}_{40}\right]^{5-}$

$\mathrm{T}_{\text {onset }}=200^{\circ} \mathrm{C}$

FTIR, TGA-DSC, XPS

50

$(\mathrm{Bmim})_{3} \mathrm{PW}_{6} \mathrm{Mo}_{6} \mathrm{O}_{40}$

$\left[\mathrm{PW}_{6} \mathrm{Mo}_{6} \mathrm{O}_{40}\right]^{3-}$

$\mathrm{T}_{\text {onset }}=300^{\circ} \mathrm{C}$ 
Table S3. Characterization of properties and structure of metal complex ILs.

\begin{tabular}{|c|c|c|c|c|}
\hline MILs & Structure & Properties & Methods & Ref. \\
\hline $\begin{array}{l}\mathrm{Cu}\left\{\mathrm{NH}_{2} \mathrm{CH}_{2} \mathrm{CH}_{2} \mathrm{OH}\right\}_{6} \\
{\left[\mathrm{CH}_{3}\left(\mathrm{CH}_{2}\right)_{3} \mathrm{CH}\left(\mathrm{C}_{2} \mathrm{H}_{5}\right) \mathrm{CO}_{2}\right]_{2}}\end{array}$ & {$\left[\mathrm{Cu}\left\{\mathrm{NH}_{2} \mathrm{CH}_{2} \mathrm{CH}_{2} \mathrm{OH}\right\}_{6}\right]^{2+}$} & $\begin{array}{l}\eta=3383 \mathrm{cP}, \sigma=0.045 \mathrm{mS} / \mathrm{cm}, \mathrm{T}_{\mathrm{d}}=190^{\circ} \mathrm{C}, \triangle \mathrm{E}=244 \mathrm{mV}, \\
\text { magnetism }\end{array}$ & ${ }^{13} \mathrm{C}$ NMR, FTIR, TGA-DSC, CV & 51 \\
\hline $\begin{array}{l}\mathrm{Cu}\left\{\mathrm{NH}_{2}\left(\mathrm{CH}_{2} \mathrm{CH}_{2} \mathrm{OH}\right)_{2}\right\}_{6} \\
{\left[\mathrm{CH}_{3}\left(\mathrm{CH}_{2}\right)_{3} \mathrm{CH}\left(\mathrm{C}_{2} \mathrm{H}_{5}\right) \mathrm{CO}_{2}\right]_{2}}\end{array}$ & {$\left[\mathrm{Cu}\left\{\mathrm{NH}_{2}\left(\mathrm{CH}_{2} \mathrm{CH}_{2} \mathrm{OH}\right)_{2}\right\}_{6}\right]^{2+}$} & $\begin{array}{l}\eta=12313 \mathrm{cP}, \sigma=0.014 \mathrm{mS} / \mathrm{cm}, \mathrm{T}_{\mathrm{d}}=230^{\circ} \mathrm{C}, \triangle \mathrm{E}=522 \mathrm{mV} \text {, } \\
\text { magnetism }\end{array}$ & & \\
\hline $\begin{array}{l}\mathrm{Cu}\left\{\mathrm{NH}_{2}\left(\mathrm{CH}_{2} \mathrm{CH}_{2} \mathrm{OH}\right)_{2}\right\}_{6} \\
{\left[\mathrm{CF}_{3} \mathrm{SO}_{3}\right]_{2}}\end{array}$ & {$\left[\mathrm{Cu}\left\{\mathrm{NH}_{2}\left(\mathrm{CH}_{2} \mathrm{CH}_{2} \mathrm{OH}\right)_{2}\right\}_{6}\right]^{2+}$} & $\begin{array}{l}\eta=1295 \mathrm{cP}, \sigma=0.067 \mathrm{mS} / \mathrm{cm}, \mathrm{T}_{\mathrm{d}}=240{ }^{\circ} \mathrm{C}, \triangle \mathrm{E}=566 \mathrm{mV} \\
\text { magnetism }\end{array}$ & & \\
\hline $\begin{array}{l}\mathrm{Cu}\left\{\mathrm{NH}_{2}\left(\mathrm{CH}_{2} \mathrm{CH}_{2} \mathrm{OH}\right)_{2}\right\}_{6} \\
{\left[\left(\mathrm{CF}_{3} \mathrm{SO}_{2}\right)_{2} \mathrm{~N}\right]_{2}}\end{array}$ & {$\left[\mathrm{Cu}\left\{\mathrm{NH}_{2}\left(\mathrm{CH}_{2} \mathrm{CH}_{2} \mathrm{OH}\right)_{2}\right\}_{6}\right]^{2+}$} & $\begin{array}{l}\eta=13900 \mathrm{cP}, \sigma=30.2 \mathrm{mS} / \mathrm{cm}, \mathrm{T}_{\mathrm{d}}=255^{\circ} \mathrm{C}, \triangle \mathrm{E}=207 \mathrm{mV} \\
\text { magnetism }\end{array}$ & & \\
\hline $\begin{array}{l}\mathrm{Mn}\left\{\mathrm{NH}_{2}\left(\mathrm{CH}_{2} \mathrm{CH}_{2} \mathrm{OH}\right)_{2}\right\}_{6} \\
{\left[\mathrm{CF}_{3} \mathrm{SO}_{3}\right]_{2}}\end{array}$ & {$\left[\mathrm{Mn}\left\{\mathrm{NH}_{2}\left(\mathrm{CH}_{2} \mathrm{CH}_{2} \mathrm{OH}\right)_{2}\right\}_{6}\right]^{2+}$} & $\begin{array}{l}\eta=11671 \mathrm{cP}, \sigma=624 \mathrm{mS} / \mathrm{cm}, \mathrm{T}_{\mathrm{d}}=215^{\circ} \mathrm{C}, \triangle \mathrm{E}=58 \mathrm{mV} \\
\text { magnetism }\end{array}$ & & \\
\hline $\begin{array}{l}\mathrm{Zn}\left\{\mathrm{NH}_{2} \mathrm{CH}_{2} \mathrm{CH}_{2} \mathrm{OH}\right\}_{6} \\
{\left[\mathrm{CF}_{3} \mathrm{SO}_{3}\right]_{2}}\end{array}$ & $\left.\left[\mathrm{Zn}\left\{\mathrm{NH}_{2} \mathrm{CH}_{2} \mathrm{CH}_{2} \mathrm{OH}\right\}\right]_{6}\right]^{2+}$ & $\begin{array}{l}\eta=2533 \mathrm{cP}, \sigma=0.0341 \mathrm{mS} / \mathrm{cm}, \mathrm{T}_{\mathrm{d}}=155^{\circ} \mathrm{C}, \triangle \mathrm{E}=335 \mathrm{mV} \text {, } \\
\text { magnetism }\end{array}$ & & \\
\hline $\begin{array}{l}\mathrm{Fe}\left\{\mathrm{NH}_{2}\left(\mathrm{CH}_{2} \mathrm{CH}_{2} \mathrm{OH}\right)_{2}\right\}_{6} \\
{\left[\mathrm{CF}_{3} \mathrm{SO}_{3}\right]_{3}}\end{array}$ & {$\left[\mathrm{Fe}\left\{\mathrm{NH}_{2}\left(\mathrm{CH}_{2} \mathrm{CH}_{2} \mathrm{OH}\right)_{2}\right\}_{6}\right]^{3+}$} & $\eta=4482 \mathrm{cP}, \sigma=0.207 \mathrm{mS} / \mathrm{cm}, \mathrm{T}_{\mathrm{d}}=260^{\circ} \mathrm{C}$ & TGA-DSC, FTIR, Raman, UV-vis, CV & 52 \\
\hline$\left[\mathrm{N}_{4444}\right]\left[\mathrm{Co}\left(\mathrm{F}_{6}-\mathrm{acac}\right)_{3}\right]$ & {$\left[\mathrm{Co}\left(\mathrm{F}_{6}-\mathrm{acac}\right)_{3}\right]^{-}$} & Hydrophobicity, $\mathrm{T}_{\mathrm{m}}=73^{\circ} \mathrm{C}, \mathrm{T}_{\mathrm{d}}=279^{\circ} \mathrm{C}$ & TGA & 53 \\
\hline$\left[\mathrm{N}_{4444}\right]\left[\mathrm{Mn}\left(\mathrm{F}_{6}-\mathrm{acac}\right)_{3}\right]$ & {$\left[\mathrm{Mn}\left(\mathrm{F}_{6}-\mathrm{acac}\right)_{3}\right]^{-}$} & Hydrophobicity, $\mathrm{T}_{\mathrm{m}}=66^{\circ} \mathrm{C}, \mathrm{T}_{\mathrm{d}}=325^{\circ} \mathrm{C}$ & & \\
\hline$\left[\mathrm{N}_{4444}\right]\left[\mathrm{Cu}\left(\mathrm{F}_{6}-\mathrm{acac}\right)_{3}\right]$ & {$\left[\mathrm{Cu}\left(\mathrm{F}_{6}-\mathrm{acac}\right)_{3}\right]^{-}$} & Hydrophobicity, $\mathrm{T}_{\mathrm{m}}=68^{\circ} \mathrm{C}, \mathrm{T}_{\mathrm{d}}=256^{\circ} \mathrm{C}$ & & \\
\hline$\left[\mathrm{N}_{1116}\right]\left[\mathrm{Co}\left(\mathrm{F}_{6}-\mathrm{acac}\right)_{3}\right]$ & {$\left[\mathrm{Co}\left(\mathrm{F}_{6}-\mathrm{acac}\right)_{3}\right]^{-}$} & Hydrophobicity, $\mathrm{T}_{\mathrm{m}}=71^{\circ} \mathrm{C}, \mathrm{T}_{\mathrm{d}}=286^{\circ} \mathrm{C}$ & & \\
\hline$\left[\mathrm{C}_{14} \mathrm{Py}\right]\left[\mathrm{Co}\left(\mathrm{F}_{6}-\mathrm{acac}\right)_{3}\right]$ & {$\left[\mathrm{Co}\left(\mathrm{F}_{6}-\mathrm{acac}\right)_{3}\right]^{-}$} & Hydrophobicity, $\mathrm{T}_{\mathrm{m}}=42{ }^{\circ} \mathrm{C}, \mathrm{T}_{\mathrm{d}}=334^{\circ} \mathrm{C}$ & & \\
\hline$\left[\mathrm{C}_{14} \mathrm{Py}\right]\left[\mathrm{Mn}\left(\mathrm{F}_{6}-\mathrm{acac}\right)_{3}\right]$ & {$\left[\mathrm{Mn}\left(\mathrm{F}_{6}-\mathrm{acac}\right)_{3}\right]^{-}$} & Hydrophobicity, $\mathrm{T}_{\mathrm{m}}=36{ }^{\circ} \mathrm{C}, \mathrm{T}_{\mathrm{d}}=298^{\circ} \mathrm{C}$ & & \\
\hline$\left[\mathrm{C}_{14} \mathrm{Py}\right]\left[\mathrm{Cu}\left(\mathrm{F}_{6}-\mathrm{acac}\right)_{3}\right]$ & {$\left[\mathrm{Cu}\left(\mathrm{F}_{6}-\mathrm{acac}\right)_{3}\right]^{-}$} & Hydrophobicity, $\mathrm{T}_{\mathrm{m}}=38^{\circ} \mathrm{C}, \mathrm{T}_{\mathrm{d}}=259^{\circ} \mathrm{C}$ & & \\
\hline$\left[\mathrm{C}_{10 \mathrm{mim}}\right]\left[\mathrm{Co}\left(\mathrm{F}_{6}-\mathrm{acac}\right)_{3}\right]$ & {$\left[\mathrm{Co}\left(\mathrm{F}_{6}-\mathrm{acac}\right)_{3}\right]^{-}$} & Hydrophobicity, $\mathrm{T}_{\mathrm{m}}<\mathrm{RT}, \mathrm{T}_{\mathrm{d}}=279^{\circ} \mathrm{C}$ & & \\
\hline$\left[\mathrm{C}_{10} \operatorname{mim}\right]\left[\mathrm{Mn}\left(\mathrm{F}_{6}-\mathrm{acac}\right)_{3}\right]$ & {$\left[\mathrm{Mn}\left(\mathrm{F}_{6}-\mathrm{acac}\right)_{3}\right]^{-}$} & Hydrophobicity, $\mathrm{T}_{\mathrm{m}}<\mathrm{RT}, \mathrm{T}_{\mathrm{d}}=263^{\circ} \mathrm{C}$ & & \\
\hline$\left[\mathrm{C}_{10} \mathrm{mim}\right]\left[\mathrm{Ni}\left(\mathrm{F}_{6}-\mathrm{acac}\right)_{3}\right]$ & {$\left[\mathrm{Ni}\left(\mathrm{F}_{6}-\mathrm{acac}\right)_{3}\right]^{-}$} & Hydrophobicity, $\mathrm{T}_{\mathrm{m}}<\mathrm{RT}, \mathrm{T}_{\mathrm{d}}=321^{\circ} \mathrm{C}$ & & \\
\hline$\left[\mathrm{C}_{10} \mathrm{mim}\right]\left[\mathrm{Cu}\left(\mathrm{F}_{6}-\mathrm{acac}\right)_{3}\right]$ & {$\left[\mathrm{Cu}\left(\mathrm{F}_{6}-\mathrm{acac}\right)_{3}\right]^{-}$} & Hydrophobicity, $\mathrm{T}_{\mathrm{m}}<\mathrm{RT}, \mathrm{T}_{\mathrm{d}}=227^{\circ} \mathrm{C}$ & & \\
\hline$\left[\mathrm{C}_{10} \mathrm{mim}\right]\left[\mathrm{Co}\left(\mathrm{F}_{3}-\mathrm{phbdo}\right)_{3}\right]$ & {$\left[\mathrm{Co}\left(\mathrm{F}_{3}-\mathrm{phbdo}\right)_{3}\right]^{-}$} & Hydrophobicity, $\mathrm{T}_{\mathrm{m}}<\mathrm{RT}, \mathrm{T}_{\mathrm{d}}=312^{\circ} \mathrm{C}$ & & \\
\hline
\end{tabular}


Table S3. Continued.

\begin{tabular}{|c|c|c|c|c|}
\hline$\left[\mathrm{C}_{10 \mathrm{mim}}\right]\left[\mathrm{Ni}\left(\mathrm{F}_{3}-\mathrm{phbdo}\right)_{3}\right]$ & {$\left[\mathrm{Ni}\left(\mathrm{F}_{3}-\mathrm{phbdo}\right)_{3}\right]^{-}$} & Hydrophobicity, $\mathrm{T}_{\mathrm{m}}<\mathrm{RT}, \mathrm{T}_{\mathrm{d}}=267^{\circ} \mathrm{C}$ & & \\
\hline$\left[\mathrm{C}_{10} \mathrm{mim}\right]\left[\mathrm{Mn}\left(\mathrm{F}_{3}-\mathrm{phbdo}\right)_{3}\right]$ & {$\left[\mathrm{Mn}\left(\mathrm{F}_{3}-\mathrm{phbdo}\right)_{3}\right]^{-}$} & Hydrophobicity, $\mathrm{T}_{\mathrm{m}}<\mathrm{RT}, \mathrm{T}_{\mathrm{d}}=255^{\circ} \mathrm{C}$ & & \\
\hline$\left[\mathrm{Pph}_{3,4}\right]\left[\mathrm{Co}\left(\mathrm{F}_{6}-\mathrm{acac}\right)_{3}\right]$ & {$\left[\mathrm{Co}\left(\mathrm{F}_{6}-\mathrm{acac}\right)_{3}\right]^{-}$} & Hydrophobicity, $\mathrm{T}_{\mathrm{m}}=73{ }^{\circ} \mathrm{C}, \mathrm{T}_{\mathrm{d}}=320^{\circ} \mathrm{C}$ & & \\
\hline$\left[\mathrm{P}_{66614}\right]\left[\mathrm{Co}(\mathrm{hfacac})_{3}\right]$ & {$\left[\mathrm{Co}(\mathrm{hfacac})_{3}\right]^{-}$} & Hydrophobicity, thermal stability & TG, silica capilary & 9 \\
\hline$\left[\mathrm{P}_{66614}\right][\mathrm{Nd}(\mathrm{hfacac}) 4]$ & {$[\mathrm{Nd}(\mathrm{hfacac}) 4]^{-}$} & & & \\
\hline$\left[\mathrm{Fe}(\mathrm{bpy})_{3}\right]_{\left[\mathrm{Tf}_{2} \mathrm{~N}\right]_{2}}$ & {$\left[\mathrm{Fe}(\mathrm{bpy})_{3}\right]^{2+}$} & $3-9 \times 10^{-7} \mathrm{~cm}^{2} / \mathrm{s}$ diffusion coefficient, $2-6 \times 10^{-3} \mathrm{~cm}^{2} / \mathrm{s}$ rate & $\mathrm{CV}$ & 54 \\
\hline$\left[\mathrm{Fe}(\text { phen })_{3}\right]\left[\mathrm{Tf}_{2} \mathrm{~N}\right]_{2}$ & {$\left[\mathrm{Fe}(\text { phen })_{3}\right]^{2+}$} & constants in $[\mathrm{Bmim}]\left[\mathrm{Tf}_{2} \mathrm{~N}\right]$ & & \\
\hline$\left[\mathrm{Fe}(\text { terpy })_{2}\right]\left[\mathrm{Tf}_{2} \mathrm{~N}\right]_{2}$ & {$\left[\mathrm{Fe}(\text { terpy })_{2}\right]^{2+}$} & & & \\
\hline $\mathrm{Fe}^{\mathrm{IIII}}\left(\mathrm{F}_{20} \mathrm{TPP}\right) \mathrm{Cl}$ & {$\left[\mathrm{Fe}^{\mathrm{III}}\left(\mathrm{F}_{20} \mathrm{TPP}\right)\right]^{+}$} & Oxygen activation & $\mathrm{CV}$, UV-vis & 55 \\
\hline $\mathrm{Fe}^{\mathrm{III}}(\mathrm{TPP}) \mathrm{OTf}$ & {$\left[\mathrm{Fe}^{\mathrm{III}}(\mathrm{TPP})\right]^{+}$} & Oxygen activation & $\mathrm{CV}$, UV-vis & 56 \\
\hline$[\mathrm{K}(\mathrm{TX}-7)][\mathrm{SCN}]$ & {$[\mathrm{K}(\mathrm{TX}-7)]^{+}$} & $\delta=8.05 \mu \mathrm{S} / \mathrm{cm}, \eta=20210 \mathrm{cP}$ & FTIR, TG & 57 \\
\hline$[\mathrm{Na}(\mathrm{TX}-7)][\mathrm{SCN}]$ & {$[\mathrm{Na}(\mathrm{TX}-7)]^{+}$} & $\delta=5.19 \mu \mathrm{S} / \mathrm{cm}, \eta=26590 \mathrm{cP}, \mathrm{T}_{\mathrm{d}}=284^{\circ} \mathrm{C}$ & & \\
\hline$[\mathrm{Li}(\mathrm{TX}-7)][\mathrm{SCN}]$ & {$[\mathrm{Li}(\mathrm{TX}-7)]^{+}$} & $\delta=5.06 \mu \mathrm{S} / \mathrm{cm}, \eta=20940 \mathrm{cP}$ & & \\
\hline$[\mathrm{Li}(\mathrm{TX}-4)][\mathrm{SCN}]$ & {$[\mathrm{Li}(\mathrm{TX}-4)]^{+}$} & $\delta=1.09 \mu \mathrm{S} / \mathrm{cm}, \eta=135900 \mathrm{cP}$ & & \\
\hline$[\mathrm{Li}(\mathrm{TX}-10)][\mathrm{SCN}]$ & {$[\mathrm{Li}(\mathrm{TX}-10)]^{+}$} & $\delta=9.71 \mu \mathrm{S} / \mathrm{cm}, \eta=7260 \mathrm{cP}$ & & \\
\hline$[\mathrm{Na}(\mathrm{TX}-10)][\mathrm{Tf} 2 \mathrm{~N}]$ & {$[\mathrm{Na}(\mathrm{TX}-10)]^{+}$} & $\delta=27.60 \mu \mathrm{S} / \mathrm{cm}, \eta=3307 \mathrm{cP}$ & & \\
\hline$[\mathrm{Na}(\mathrm{TX}-10)][\mathrm{SCN}]$ & {$[\mathrm{Na}(\mathrm{TX}-10)]^{+}$} & $\delta=14.66 \mu \mathrm{S} / \mathrm{cm}, \eta=8680 \mathrm{cP}$ & & \\
\hline$[\mathrm{Na}(\mathrm{TX}-10)][\mathrm{PhO}]$ & {$[\mathrm{Na}(\mathrm{TX}-10)]^{+}$} & $\delta=3.64 \mu \mathrm{S} / \mathrm{cm}, \eta=4410 \mathrm{cP}$ & & \\
\hline$[\mathrm{Na}(\mathrm{TX}-10)][\mathrm{Im}]$ & {$[\mathrm{Na}(\mathrm{TX}-10)]^{+}$} & $\delta=3.32 \mu \mathrm{S} / \mathrm{cm}, \eta=11010 \mathrm{cP}$ & & \\
\hline
\end{tabular}


Table S4. Oxidative desulfurization of organic sulfide in metal chloride ILs over the past decade.

\begin{tabular}{|c|c|c|c|c|c|c|}
\hline Catalyst & Extractant & Oxidant & $\begin{array}{l}\text { Oil/Sulfur } \\
\text { compounds }\end{array}$ & Reaction conditions & $\begin{array}{l}\text { S- } \\
\text { removal/\% }\end{array}$ & Ref. \\
\hline \multirow[t]{2}{*}[\mathrm{C}_{4}\mathrm{mim}]{$\mathrm{Cl} / \mathrm{ZnCl}_{2}$} & {$\left[\mathrm{C}_{4} \mathrm{mim}\right] \mathrm{Cl} / \mathrm{ZnCl}_{2}$} & $\mathrm{H}_{2} \mathrm{O}_{2}$ & n-octane/DBT & $\begin{array}{l}\mathrm{T}=90 \quad{ }^{\circ} \mathrm{C} ; \mathrm{t}=60 \quad \mathrm{~min} ; \mathrm{n}(\mathrm{O}) / \mathrm{n}(\mathrm{S})=8: 1 \\
\mathrm{~m}(\mathrm{MIL}) / \mathrm{m}(\text { oil })=1: 5 ; 6 \text { recycles }\end{array}$ & 98.4 & 58 \\
\hline & & & Diesel fuel/DBT & $\begin{array}{l}\mathrm{T}=90^{\circ} \mathrm{C} ; \mathrm{t}=60 \mathrm{~min} ; \text { Initial S-content } 64 \mathrm{ppm} \\
\mathrm{m}(\mathrm{MIL})=3 \mathrm{~g} ; \mathrm{m}(\mathrm{O})=0.136 \mathrm{~g} ; \mathrm{n}(\mathrm{O}) / \mathrm{n}(\mathrm{S})=8: 1 \\
\mathrm{~m}(\mathrm{MIL}) / \mathrm{m}(\mathrm{oil})=1: 5 ; 6 \text { recycles }\end{array}$ & 87.7 & \\
\hline \multirow[t]{2}{*}[\mathrm{C}_{4}\mathrm{mim}]{$\mathrm{Cl} / 3 \mathrm{ZnCl}_{2}$} & {$\left[\mathrm{C}_{4} \mathrm{mim}\right] \mathrm{Cl} / 3 \mathrm{ZnCl}_{2}$} & $\mathrm{H}_{2} \mathrm{O}_{2}$ & n-octane/DBT & $\begin{array}{l}\mathrm{T}=45{ }^{\circ} \mathrm{C} ; \mathrm{t}=180 \mathrm{~min} ; \mathrm{n}(\mathrm{O}) / \mathrm{n}(\mathrm{S})=8: 1 \\
\mathrm{~m}(\mathrm{MIL}) / \mathrm{m}(\text { oil })=1: 2 ; 7 \text { recycles }\end{array}$ & 99.9 & 59 \\
\hline & & & FCC diesel fuel/DBT & $\begin{array}{l}\mathrm{T}=45^{\circ} \mathrm{C} ; \mathrm{t}=180 \mathrm{~min} ; \text { Initial S-content } 460 \mathrm{ppm} \\
\mathrm{n}(\mathrm{O}) / \mathrm{n}(\mathrm{S})=8: 1 ; \mathrm{m}(\mathrm{MIL}) / \mathrm{m}(\text { oil })=1: 2 ; 5 \text { recycles }\end{array}$ & 63.5 & \\
\hline$\left[\mathrm{C}_{4} \mathrm{mim}\right] \mathrm{Cl} / \mathrm{AlCl}_{3}$ & {$[\mathrm{Omim}] \mathrm{BF}_{4}$} & $\begin{array}{l}\mathrm{H}_{2} \mathrm{O}_{2} \text { and } \\
\text { formic acid }\end{array}$ & $\begin{array}{l}\text { Gasoline/BT, TP, 2- } \\
\text { MT, 3-MT }\end{array}$ & $\begin{array}{l}\mathrm{T}=50^{\circ} \mathrm{C} ; \mathrm{t}=60 \mathrm{~min} ; \text { Initial S-content } 1400 \mathrm{ppm} ; \\
\mathrm{V}(\text { extractant }): \mathrm{V}(\mathrm{oil})=1: 1\end{array}$ & 97 & 60 \\
\hline \multirow[t]{2}{*}[\mathrm{C}_{4}\mathrm{mim}]{$\mathrm{Cl} / \mathrm{FeCl}_{3}$} & {$\left[\mathrm{C}_{4} \mathrm{mim}\right] \mathrm{Cl} / \mathrm{FeCl}_{3}$} & $\mathrm{H}_{2} \mathrm{O}_{2}$ & $\begin{array}{l}\text { n-octane/DBT, BT, TS, } \\
\text { 4,6-DMDBT, 3-MT }\end{array}$ & $\begin{array}{l}\mathrm{T}=30^{\circ} \mathrm{C} ; \mathrm{t}=40 \mathrm{~min} ; \text { Initial S-content } 500 \mathrm{ppm} ; \\
\mathrm{m}(\mathrm{MIL}): \mathrm{m}(\mathrm{oil})=1: 1\end{array}$ & 92 & 61 \\
\hline & & & n-octane/DBT & $\begin{array}{l}\mathrm{T}=30^{\circ} \mathrm{C} ; \mathrm{t}=40 \mathrm{~min} ; \text { Initial S-content } 500 \mathrm{ppm} ; \\
\mathrm{m}(\mathrm{MIL}): \mathrm{m}(\text { oil })=1: 1 ; \text { ultrasonic power } 250 \mathrm{~W}\end{array}$ & 96.5 & 61 \\
\hline$\left[\mathrm{C}_{4} \mathrm{mim}\right] \mathrm{Cl} / \mathrm{CoCl}_{2}$ & {$\left[\mathrm{C}_{4} \mathrm{mim}\right] \mathrm{Cl} / \mathrm{CoCl}_{2}$} & $\begin{array}{l}\text { Potassium } \\
\text { monopersulfate }\end{array}$ & n-octane/BT & $\begin{array}{l}\mathrm{T}=46^{\circ} \mathrm{C} ; \mathrm{t}=23 \mathrm{~min} ; \mathrm{m}(\mathrm{MIL})=3.2 \mathrm{~g} ; \mathrm{m}(\mathrm{O})=1.6 \\
\mathrm{~g}\end{array}$ & 95.4 & 62 \\
\hline diperiodatocuprate(III) & {$\left[\mathrm{C}_{4} \mathrm{mim}\right] \mathrm{Cl} / \mathrm{FeCl}_{3}$} & air & $\begin{array}{l}\text { Diesel fuel/DBT, BT, } \\
\text { 4,6-DMDBT }\end{array}$ & $\begin{array}{l}\mathrm{T}=25^{\circ} \mathrm{C} ; \mathrm{t}=10 \mathrm{~min} ; \text { Initial S-content } 200 \mathrm{ppm} ; \\
\mathrm{W}(\text { catalyst })=1.25 \mathrm{wt} \% ; \mathrm{V}(\mathrm{MIL}) / \mathrm{V}(\mathrm{oil})=1: 4 ; \mathrm{U}=16 \\
\mathrm{kV} ; \mathrm{f}=79 \mathrm{kHz} ; 4 \text { recycles }\end{array}$ & 97.5 & 63 \\
\hline \multirow[t]{2}{*}{ Schiff-base IL } & {$\left[\mathrm{C}_{4} \mathrm{mim}\right] \mathrm{Cl} / \mathrm{FeCl}_{3}$} & $\mathrm{O}_{2}$ & n-octone/TS & $\begin{array}{l}\mathrm{T}=62^{\circ} \mathrm{C} ; \mathrm{t}=360 \mathrm{~min} ; \text { oxygen flow rate } 50 \mathrm{~mL} / \mathrm{min} \\
\mathrm{V}(\mathrm{IL}): \mathrm{V}(\text { oil })=8: 25 ; \mathrm{m}(\mathrm{CoL})=0.13 \mathrm{~g} ; 5 \text { recycles }\end{array}$ & 96 & 64 \\
\hline & & & Diesel fuel/TS & $\begin{array}{l}\mathrm{T}=62{ }^{\circ} \mathrm{C} ; \mathrm{t}=240 \mathrm{~min} ; \text { Initial S-content } 472.44 \\
\mu \mathrm{g} / \mathrm{g} ; \text { oxygen flow rate } 50 \mathrm{~mL} / \mathrm{min} ; \mathrm{V}(\mathrm{IL}): \mathrm{V}(\text { oil })= \\
8: 25 ; \mathrm{m}(\mathrm{CoL})=0.13 \mathrm{~g}\end{array}$ & 100 & \\
\hline$\left[\mathrm{C}_{5} \mathrm{mim}\right] \mathrm{Cl} / \mathrm{CoCl}_{2}$ & {$\left[\mathrm{C}_{5} \mathrm{mim}\right] \mathrm{Cl} / \mathrm{CoCl}_{2}$} & {$\left[\mathrm{C}_{5} \mathrm{mim}\right]_{2}\left[\mathrm{~S}_{2} \mathrm{O}_{8}\right]$} & $\begin{array}{l}\text { n-octane/DBT, } \\
\text { 4,6-DMDBT }\end{array}$ & $\begin{array}{l}\mathrm{T}=30^{\circ} \mathrm{C} ; \mathrm{t}=65 \mathrm{~min} ; \text { Initial S-content } 500 \mathrm{ppm} ; \\
\mathrm{m}(\mathrm{O})=0.5 \mathrm{~g} ; \mathrm{m}(\mathrm{MIL}): \mathrm{m}(\text { oil })=1: 4 ; 4 \text { recycles }\end{array}$ & 98.7 & 65 \\
\hline \multirow[t]{2}{*}[\mathrm{Omim}]{$\mathrm{Cl} / \mathrm{FeCl}_{3}$} & {$[\mathrm{Omim}] \mathrm{Cl} / \mathrm{FeCl}_{3}$} & $\mathrm{H}_{2} \mathrm{O}_{2}$ & $\begin{array}{l}\text { n-octane/DBT, } \\
\text { 4,6-DMDBT }\end{array}$ & $\begin{array}{l}\mathrm{T}=25^{\circ} \mathrm{C} ; \mathrm{t}=15 \mathrm{~min} ; \text { Initial S-content } 1000 \mathrm{ppm} \\
\mathrm{n}(\mathrm{O}): \mathrm{n}(\mathrm{S})=5: 1 ; \mathrm{V}(\mathrm{MIL}): \mathrm{V}(\text { oil })=1: 10 ; 8 \text { recycles }\end{array}$ & 100 & 19 \\
\hline & & & Diesel fuel & $\begin{array}{l}\mathrm{T}=25^{\circ} \mathrm{C} ; \mathrm{t}=15 \mathrm{~min} ; \text { Initial S-content } 590 \mathrm{ppm} ; \\
\mathrm{n}(\mathrm{O}): \mathrm{n}(\mathrm{S})=5: 1 ; \mathrm{V}(\mathrm{MIL}): \mathrm{V}(\text { oil })=1: 10\end{array}$ & 85 & 19 \\
\hline$[\mathrm{Amim}] \mathrm{Cl} / \mathrm{FeCl}_{3}$ & {$[\mathrm{Amim}] \mathrm{Cl} / \mathrm{FeCl}_{3}$} & $\mathrm{H}_{2} \mathrm{O}_{2}$ & n-octane/DBT, BT, TS & $\begin{array}{l}\mathrm{T}=25 \quad{ }^{\circ} \mathrm{C} ; \mathrm{t}=20 \mathrm{~min} ; \mathrm{n}(\mathrm{O}) / \mathrm{n}(\mathrm{S})=8: 1 \\
\mathrm{~V}(\mathrm{MIL}): \mathrm{V}(\mathrm{oil})=1: 3 ; 2 \text { recycles }\end{array}$ & 96.96 & 66 \\
\hline$\left[\mathrm{C}_{4}(\mathrm{mim})_{2}\right] \mathrm{Cl}_{2} / 2 \mathrm{FeCl}_{3}$ & acetonitrile & $\mathrm{H}_{2} \mathrm{O}_{2}$ & n-octane/DBT & $\begin{array}{l}\mathrm{T}=40^{\circ} \mathrm{C} ; \mathrm{t}=10 \mathrm{~min} ; \text { Initial S-content } 500 \mathrm{ppm} \\
\mathrm{n}(\mathrm{O}): \mathrm{n}(\mathrm{S})=6: 1 ; \mathrm{m}(\mathrm{MIL})=20 \mathrm{mg} \\
\mathrm{V}(\text { extractant }): \mathrm{V}(\mathrm{oil})=1: 5 ; 6 \text { recycles }\end{array}$ & 97.07 & 18 \\
\hline$\left[\left(\mathrm{CH}_{2}\right)_{4} \mathrm{SO}_{3} \mathrm{HMIm}\right] \mathrm{Cl} / \mathrm{ZnCl}_{2}$ & {$\left[\left(\mathrm{CH}_{2}\right)_{4} \mathrm{SO}_{3} \mathrm{HMIm}\right] \mathrm{Cl} / \mathrm{ZnCl}_{2}$} & $\mathrm{H}_{2} \mathrm{O}_{2}$ & Diesel fuel & $\begin{array}{l}\mathrm{T}=60{ }^{\circ} \mathrm{C} ; \mathrm{t}=180 \mathrm{~min} ; \text { Initial S-content } 225 \mathrm{ppm} ; \\
\mathrm{n}(\mathrm{O}): \mathrm{n}(\mathrm{S})=30: 1 ; \mathrm{m}(\mathrm{MIL}): \mathrm{m}(\mathrm{oil})=1: 2 ; 5 \text { recycles }\end{array}$ & 40.7 & 67 \\
\hline
\end{tabular}


Table S4. Continued.

\begin{tabular}{|c|c|c|c|c|c|c|c|}
\hline$\left[\left(\mathrm{CH}_{2}\right)_{2} \mathrm{COOHmim}\right] \mathrm{Cl} / \mathrm{FeCl}_{3}$ & {$\left[\left(\mathrm{CH}_{2}\right)_{2} \mathrm{COOHmim}\right] \mathrm{Cl} / \mathrm{FeCl}_{3}$} & $\mathrm{H}_{2} \mathrm{O}_{2}$ & \multicolumn{2}{|c|}{ n-octane/DBT, BT, TS } & $\begin{array}{l}\mathrm{T}=25 \quad{ }^{\circ} \mathrm{C} ; \mathrm{t}=20 \quad \min ; \mathrm{n}(\mathrm{O}) / \mathrm{n}(\mathrm{S})=4: 1 \\
\mathrm{~V}(\mathrm{M} I \mathrm{~L}): \mathrm{V}(\mathrm{oil})=1: 3\end{array}$ & 97.1 & 66 \\
\hline$\left[\left(\mathrm{CH}_{2}\right)_{2} \mathrm{COOHmim}\right] \mathrm{Cl} / \mathrm{ZnCl}_{2}$ & {$\left[\left(\mathrm{CH}_{2}\right)_{2} \mathrm{COOHmim}\right] \mathrm{Cl} / \mathrm{ZnCl}_{2}$} & $\mathrm{H}_{2} \mathrm{O}_{2}$ & \multicolumn{2}{|c|}{ n-octane/DBT, BT, TS } & $\begin{array}{l}\mathrm{T}=60{ }^{\circ} \mathrm{C} ; \mathrm{t}=240 \mathrm{~min} ; \mathrm{n}(\mathrm{O}) / \mathrm{n}(\mathrm{S})=8: 1 \\
\mathrm{~V}(\mathrm{MIL}): \mathrm{V}(\mathrm{oil})=1: 3\end{array}$ & 99.62 & 66 \\
\hline \multirow[t]{2}{*}[(\mathrm{C}_{8}\mathrm{H}_{17})_{3}\mathrm{CH}_{3}\mathrm{N}]{$\mathrm{Cl} / \mathrm{FeCl}_{3}$} & {$\left[\left(\mathrm{C}_{8} \mathrm{H}_{17}\right)_{3} \mathrm{CH}_{3} \mathrm{~N}\right] \mathrm{Cl} / \mathrm{FeCl}_{3}$} & $\mathrm{H}_{2} \mathrm{O}_{2}$ & $\begin{array}{l}\text { n-octane/BT, } \\
\text { 4,6-DMDBT }\end{array}$ & DBT, & $\begin{array}{l}\mathrm{T}=25^{\circ} \mathrm{C} ; \mathrm{t}=60 \mathrm{~min} ; \mathrm{n}(\mathrm{O}) / \mathrm{n}(\mathrm{S})=14: 1 ; \mathrm{V}(\mathrm{oil})=5 \\
\mathrm{~mL} ; \mathrm{n}(\mathrm{MIL})=0.702 \mathrm{mmol} ; \quad 6 \text { recycles }\end{array}$ & 97.9 & 68 \\
\hline & & & FCC gasoline & & $\begin{array}{l}\mathrm{T}=25^{\circ} \mathrm{C} ; \mathrm{t}=60 \mathrm{~min} ; \text { Initial S-content } 360 \mathrm{ppm} ; \\
\mathrm{n}(\mathrm{MIL})=1.404 \mathrm{mmol} ; \mathrm{V}(\mathrm{O})=112 \mu \mathrm{L}\end{array}$ & 69.4 & \\
\hline$\left[\left(\mathrm{C}_{4} \mathrm{H}_{9}\right)_{3} \mathrm{CH}_{3} \mathrm{~N}\right] \mathrm{Cl} / \mathrm{FeCl}_{3}$ & {$\left[\left(\mathrm{C}_{4} \mathrm{H}_{9}\right)_{3} \mathrm{CH}_{3} \mathrm{~N}\right] \mathrm{Cl} / \mathrm{FeCl}_{3}$} & $\mathrm{H}_{2} \mathrm{O}_{2}$ & $\begin{array}{l}\text { n-octane/BT, } \\
\text { 4,6-DMDBT }\end{array}$ & DBT, & $\begin{array}{l}\mathrm{T}=25^{\circ} \mathrm{C} ; \mathrm{t}=60 \mathrm{~min} ; \mathrm{n}(\mathrm{O}) / \mathrm{n}(\mathrm{S})=14: 1 ; \mathrm{V}(\text { oil })=5 \\
\mathrm{~mL} ; \mathrm{n}(\mathrm{MIL})=0.702 \mathrm{mmol}\end{array}$ & 95.8 & 68 \\
\hline$\left[\mathrm{C}_{10} \mathrm{H}_{21}\left(\mathrm{CH}_{3}\right)_{3} \mathrm{~N}\right] \mathrm{Cl} / \mathrm{FeCl}_{3}$ & {$\left[\mathrm{C}_{10} \mathrm{H}_{21}\left(\mathrm{CH}_{3}\right)_{3} \mathrm{~N}\right] \mathrm{Cl} / \mathrm{FeCl}_{3}$} & $\mathrm{H}_{2} \mathrm{O}_{2}$ & $\begin{array}{l}\text { n-octane/BT, } \\
\text { 4,6-DMDBT }\end{array}$ & DBT, & $\begin{array}{l}\mathrm{T}=25^{\circ} \mathrm{C} ; \mathrm{t}=60 \mathrm{~min} ; \mathrm{n}(\mathrm{O}) / \mathrm{n}(\mathrm{S})=14: 1 ; \mathrm{V}(\text { oil })=5 \\
\mathrm{~mL} ; \mathrm{n}(\mathrm{MIL})=0.702 \mathrm{mmol}\end{array}$ & 93.4 & 68 \\
\hline$\left[\left(\mathrm{C}_{10} \mathrm{H}_{21}\right)_{2}\left(\mathrm{CH}_{3}\right)_{2} \mathrm{~N}\right] \mathrm{Cl} / \mathrm{FeCl}_{3}$ & {$\left[\left(\mathrm{C}_{10} \mathrm{H}_{21}\right)_{2}\left(\mathrm{CH}_{3}\right)_{2} \mathrm{~N}\right] \mathrm{Cl} / \mathrm{FeCl}_{3}$} & $\mathrm{H}_{2} \mathrm{O}_{2}$ & $\begin{array}{l}\text { n-octane/BT, } \\
\text { 4,6-DMDBT }\end{array}$ & DBT, & $\begin{array}{l}\mathrm{T}=25^{\circ} \mathrm{C} ; \mathrm{t}=60 \mathrm{~min} ; \mathrm{n}(\mathrm{O}) / \mathrm{n}(\mathrm{S})=14: 1 ; \mathrm{V}(\text { oil })=5 \\
\mathrm{~mL} ; \mathrm{n}(\mathrm{MIL})=0.702 \mathrm{mmol}\end{array}$ & 98.7 & 68 \\
\hline$\left(\mathrm{C}_{2} \mathrm{H}_{5}\right)_{3} \mathrm{NHCl} / \mathrm{FeCl}_{3}$ & $\left(\mathrm{C}_{2} \mathrm{H}_{5}\right)_{3} \mathrm{NHCl} / \mathrm{FeCl}_{3}$ & $\mathrm{H}_{2} \mathrm{O}_{2}$ & n-octane/DBT & & $\begin{array}{l}\mathrm{T}=30^{\circ} \mathrm{C} ; \mathrm{t}=5 \mathrm{~min} ; \mathrm{n}(\mathrm{O}) / \mathrm{n}(\mathrm{S})=6: 1 ; \mathrm{V}(\mathrm{oil})=5 \mathrm{~mL} \\
\mathrm{~m}(\mathrm{MIL})=1.2451 \mathrm{~g} ; 6 \text { recycles }\end{array}$ & 97.1 & 69 \\
\hline$\left[\left(\mathrm{CH}_{3}\right)_{4} \mathrm{~N}\right] \mathrm{Cl} / \mathrm{FeCl}_{3}$ & {$[\mathrm{Bmim}] \mathrm{BF}_{4}$} & $\mathrm{H}_{2} \mathrm{O}_{2}$ & n-octane/DBT & & $\begin{array}{l}\mathrm{T}=30^{\circ} \mathrm{C} ; \mathrm{t}=60 \mathrm{~min} ; \mathrm{n}(\mathrm{O}) / \mathrm{n}(\mathrm{S})=11.6: 1 ; \mathrm{n}(\mathrm{O})= \\
0.907 \mathrm{mmol} ; \mathrm{V}(\mathrm{oil})=5 \mathrm{~mL} ; \mathrm{n}(\mathrm{MIL})=0.195 \mathrm{mmol} ; \\
\mathrm{V}(\text { extractant })=1 \mathrm{~mL} ; 6 \text { recycles }\end{array}$ & 97 & 70 \\
\hline$\left[\mathrm{C}_{14} \mathrm{H}_{29} \mathrm{~N}\left(\mathrm{CH}_{3}\right)_{3}\right] \mathrm{Cl} / \mathrm{FeCl}_{3}$ & {$\left[\mathrm{Bmim}_{\mathrm{B}} \mathrm{BF}_{4}\right.$} & $\mathrm{H}_{2} \mathrm{O}_{2}$ & n-octane/DBT & & $\begin{array}{l}\mathrm{T}=30^{\circ} \mathrm{C} ; \mathrm{t}=60 \mathrm{~min} ; \mathrm{n}(\mathrm{O}) / \mathrm{n}(\mathrm{S})=11.6: 1 ; \mathrm{n}(\mathrm{O})= \\
0.907 \mathrm{mmol} ; \mathrm{V}(\mathrm{oil})=5 \mathrm{~mL} ; \mathrm{n}(\mathrm{MIL})=0.195 \mathrm{mmol} ; \\
\mathrm{V}(\text { extractant })=1 \mathrm{~mL}\end{array}$ & 95.4 & 70 \\
\hline$\left[\mathrm{C}_{18} \mathrm{H}_{37} \mathrm{~N}\left(\mathrm{CH}_{3}\right)_{3}\right] \mathrm{Cl} / \mathrm{FeCl}_{3}$ & {$[\mathrm{Bmim}] \mathrm{BF}_{4}$} & $\mathrm{H}_{2} \mathrm{O}_{2}$ & n-octane/DBT & & $\begin{array}{l}\mathrm{T}=30^{\circ} \mathrm{C} ; \mathrm{t}=60 \mathrm{~min} ; \mathrm{n}(\mathrm{O}) / \mathrm{n}(\mathrm{S})=11.6: 1 ; \mathrm{n}(\mathrm{O})= \\
0.907 \mathrm{mmol} ; \mathrm{V}(\mathrm{oil})=5 \mathrm{~mL} ; \mathrm{n}(\mathrm{MIL})=0.195 \mathrm{mmol} ; \\
\mathrm{V}(\text { extractant })=1 \mathrm{~mL}\end{array}$ & 94.3 & 70 \\
\hline$\left(\mathrm{C}_{4} \mathrm{H}_{9}\right)_{4} \mathrm{NCl} / \mathrm{FeCl}_{3}$ & {$[\mathrm{Dmim}] \mathrm{BF}_{4}$} & $\mathrm{H}_{2} \mathrm{O}_{2}$ & n-octane/DBT & & $\begin{array}{l}\mathrm{T}=20^{\circ} \mathrm{C} ; \mathrm{t}=60 \min ; \mathrm{n}(\mathrm{O}): \mathrm{n}(\mathrm{S})=6: 1 ; \mathrm{m}(\mathrm{IL}): \mathrm{m}(\mathrm{oil}) \\
=1: 5 ; \mathrm{n}(\mathrm{MIL})=0.09 \mathrm{~g} ; 8 \text { recycles }\end{array}$ & 98.4 & 71 \\
\hline \multirow[t]{2}{*}{$\mathrm{C}_{5} \mathrm{H}_{9} \mathrm{NO} / \mathrm{SnCl}_{2}$} & $\mathrm{C}_{5} \mathrm{H}_{9} \mathrm{NO} / \mathrm{SnCl}_{2}$ & $\mathrm{H}_{2} \mathrm{O}_{2}$ & n-octane/DBT & & $\begin{array}{l}\mathrm{T}=30 \quad{ }^{\circ} \mathrm{C} ; \mathrm{t}=30 \mathrm{~min} ; \quad \mathrm{n}(\mathrm{O}) / \mathrm{n}(\mathrm{S})=6: 1 ; \\
\mathrm{V}(\mathrm{MIL}) / \mathrm{V}(\mathrm{oil})=1: 3 ; \quad 5 \text { recycles }\end{array}$ & 94.8 & 72 \\
\hline & & & Diesel fuel/DBT & & $\begin{array}{l}\mathrm{T}=30 \quad{ }^{\circ} \mathrm{C} ; \mathrm{t}=30 \quad \mathrm{~min} ; \quad \mathrm{n}(\mathrm{O}) / \mathrm{n}(\mathrm{S})=6: 1 \\
\mathrm{~V}(\mathrm{MIL}) / \mathrm{V}(\mathrm{oil})=1: 3\end{array}$ & 87.6 & \\
\hline \multirow[t]{2}{*}[\mathrm{C}_{6}{}^{3}\mathrm{MPy}]{$\mathrm{Cl} / \mathrm{FeCl}_{3}$} & {$\left[\mathrm{C}_{6}{ }^{3} \mathrm{MPy}\right] \mathrm{Cl} / \mathrm{FeCl}_{3}$} & $\mathrm{H}_{2} \mathrm{O}_{2}$ & n-octane/DBT & & $\begin{array}{l}\mathrm{T}=25{ }^{\circ} \mathrm{C} ; \mathrm{t}=20 \mathrm{~min} ; \mathrm{n}(\mathrm{O}) / \mathrm{n}(\mathrm{S})=4: 1 \\
\mathrm{~m}(\mathrm{MIL}) / \mathrm{m}(\text { oil })=1: 3 ; 5 \text { recycles }\end{array}$ & 100 & 73 \\
\hline & & & Gasoline/DBT & & $\begin{array}{l}\mathrm{T}=25 \quad{ }^{\circ} \mathrm{C} ; \quad \mathrm{t}=20 \quad \mathrm{~min} ; \quad \mathrm{n}(\mathrm{O}) / \mathrm{n}(\mathrm{S})=4: 1 \\
\mathrm{~m}(\mathrm{MIL}) / \mathrm{m}(\mathrm{oil})=1: 3\end{array}$ & 37.7 & \\
\hline \multirow[t]{2}{*}[\mathrm{C}_{8}{}^{3}\mathrm{MPy}]{$\mathrm{Cl} / \mathrm{FeCl}_{3}$} & {$\left[\mathrm{C}_{8}^{3} \mathrm{MPy}\right] \mathrm{Cl} / \mathrm{FeCl}_{3}$} & $\mathrm{H}_{2} \mathrm{O}_{2}$ & n-octane/DBT & & $\begin{array}{l}\mathrm{T}=25 \quad{ }^{\circ} \mathrm{C} ; \mathrm{t}=30 \mathrm{~min} ; \mathrm{n}(\mathrm{O}) / \mathrm{n}(\mathrm{S})=6: 1 \\
\mathrm{~m}(\mathrm{MIL}) / \mathrm{m}(\text { oil })=1: 5 ; 4 \text { recycles }\end{array}$ & 100 & 74 \\
\hline & & & gasoline/DBT & & $\begin{array}{l}\mathrm{T}=25^{\circ} \mathrm{C} ; \mathrm{t}=30 \mathrm{~min} ; \text { Initial S-content } 468 \mathrm{ppm} ; \\
\mathrm{n}(\mathrm{O}) / \mathrm{n}(\mathrm{S})=6: 1 ; \mathrm{m}(\mathrm{MIL}) / \mathrm{m}(\mathrm{oil})=1: 3\end{array}$ & 44.2 & \\
\hline$[\mathrm{BPy}] \mathrm{Cl} / \mathrm{FeCl}_{3}$ & {$[\mathrm{BPy}] \mathrm{Cl} / \mathrm{FeCl}_{3}$} & $\mathrm{H}_{2} \mathrm{O}_{2}$ & n-octane/DBT & & $\begin{array}{l}\mathrm{T}=40^{\circ} \mathrm{C} ; \mathrm{t}=10 \mathrm{~min} ; \mathrm{n}(\mathrm{O}) / \mathrm{n}(\mathrm{S})=8: 1 ; \text { model oil }=5 \\
\mathrm{~mL} ; \mathrm{n}(\mathrm{MIL})=0.78 \mathrm{mmol} ; 6 \text { recycles }\end{array}$ & 95.3 & 75 \\
\hline
\end{tabular}


Table S4. Continued.

\section{$\left[\mathrm{C}_{4}\right.$ mpip $] \mathrm{Cl} / \mathrm{FeCl}_{3}$}

$\mathrm{ChCl} / \mathrm{FeCl}_{3}$

$[\mathrm{Hnmp}] \mathrm{Cl} / \mathrm{ZnCl}_{2}$

$[\mathrm{Hnmp}] \mathrm{Cl} / \mathrm{FeCl}_{3}$

$\mathrm{DMSO} / \mathrm{ZnCl}_{2}$

$[\mathrm{Hnoc}] \mathrm{Cl} / 3 \mathrm{ZnCl}_{2}$

$[\mathrm{HDBN}] \mathrm{Cl} / \mathrm{ZnCl}_{2}$

$[\mathrm{HDBN}] \mathrm{Cl} / \mathrm{ZnCl}_{2}$

$[\mathrm{ODBU}] \mathrm{Cl} / 3 \mathrm{ZnCl} \mathrm{l}_{2}$

$[\mathrm{Omim}] \mathrm{BF}_{4}$

$[\mathrm{Hnmp}] \mathrm{Cl} / \mathrm{ZnCl}_{2}$

$[\mathrm{Hnmp}] \mathrm{Cl} / \mathrm{FeCl}_{3}$

$\mathrm{DMSO} / \mathrm{ZnCl}_{2}$

$[\mathrm{Hnoc}] \mathrm{Cl} / 3 \mathrm{ZnCl} 2$

[ODBU]Cl/3ZnCl

\section{n-octane/BT}

n-octane/4,6-DMDBT

$\mathrm{H}_{2} \mathrm{O}_{2}$

$\mathrm{H}_{2} \mathrm{O}_{2}$

$\mathrm{H}_{2} \mathrm{O}_{2}$

$\mathrm{H}_{2} \mathrm{O}_{2}$

$\mathrm{H}_{2} \mathrm{O}_{2}$

$\mathrm{H}_{2} \mathrm{O}_{2}$

$\mathrm{H}_{2} \mathrm{O}_{2}$

$\mathrm{H}_{2} \mathrm{O}_{2} \quad$ n-octane/DBT
n-octane/DBT

n-octane/DBT

n-octane/DBT

FCC diesel fuel

n-octane/DBT

n-octane/DBT

Diesel oil/DBT

n-octane/DBT

diesel/DBT

n-octane/DBT

diesel/DBT

diesel/DBT
$\mathrm{T}=40^{\circ} \mathrm{C} ; \mathrm{t}=10 \mathrm{~min} ; \mathrm{n}(\mathrm{O}) / \mathrm{n}(\mathrm{S})=8: 1 ;$ model oil $=5$

$\mathrm{mL} ; \mathrm{n}(\mathrm{MIL})=0.78 \mathrm{mmol}$

$\mathrm{T}=40{ }^{\circ} \mathrm{C} ; \mathrm{t}=10 \mathrm{~min} ; \mathrm{n}(\mathrm{O}) / \mathrm{n}(\mathrm{S})=8: 1 ;$ model oil $=5 \quad 54.8$ $\mathrm{mL} ; \mathrm{n}(\mathrm{MIL})=0.78 \mathrm{mmol}$

$\mathrm{T}=30{ }^{\circ} \mathrm{C} ; \mathrm{t}=60 \mathrm{~min} ; \mathrm{n}(\mathrm{O}) / \mathrm{n}(\mathrm{S})=3.5: 1 ; \quad 92.1$

$\mathrm{V}($ extractant $): \mathrm{V}($ oil $)=1: 5 ; \mathrm{n}(\mathrm{MIL})=0.078 \mathrm{mmol}$

$\mathrm{T}=30^{\circ} \mathrm{C} ; \mathrm{t}=60 \mathrm{~min} ; \mathrm{n}(\mathrm{O}) / \mathrm{n}(\mathrm{S})=6: 1 ; 97.2$

$\mathrm{V}($ extractant $): \mathrm{V}($ oil $)=1: 5 ; \mathrm{m}(\mathrm{MIL})=0.03 \mathrm{~g} ; 5$

recycles

$\mathrm{T}=75{ }^{\circ} \mathrm{C} ; \mathrm{t}=20 \mathrm{~min}$; Initial S-content $500 \mathrm{ppm} ; \quad 99.9$

$\mathrm{n}(\mathrm{O}): \mathrm{n}(\mathrm{S})=4: 1 ; \mathrm{m}(\mathrm{MIL}): \mathrm{m}($ oil $)=1: 3 ; 7$ recycles

$\mathrm{T}=75^{\circ} \mathrm{C} ; \mathrm{t}=20 \mathrm{~min}$; Initial S-content $224.6 \mathrm{ppm} ; \quad 38.2$

$\mathrm{n}(\mathrm{O}): \mathrm{n}(\mathrm{S})=50: 1 ; \mathrm{m}(\mathrm{MIL}): \mathrm{m}($ oil $)=1: 3$

$\mathrm{T}=30{ }^{\circ} \mathrm{C} ; \mathrm{t}=20 \mathrm{~min}$; Initial S-content $500 \mathrm{ppm} ; \quad 99.84$

$\mathrm{n}(\mathrm{O}): \mathrm{n}(\mathrm{S})=8: 1 ; \mathrm{V}(\mathrm{MIL}): \mathrm{V}(\mathrm{oil})=1: 10 ; 4$ recycles

$\mathrm{T}=30^{\circ} \mathrm{C} ; \mathrm{t}=40 \mathrm{~min}$; Initial S-content $1000 \mathrm{ppm} ; 78$

$\mathrm{n}(\mathrm{O}): \mathrm{n}(\mathrm{S})=6: 1 ; \mathrm{m}(\mathrm{MIL}): \mathrm{m}(\mathrm{oil})=1: 3$

$\mathrm{T}=30{ }^{\circ} \mathrm{C} ; \mathrm{t}=40 \mathrm{~min}$; Initial S-content $140 \mathrm{ppm} ; \quad 65$

$\mathrm{n}(\mathrm{O}): \mathrm{n}(\mathrm{S})=6: 1 ; \mathrm{m}(\mathrm{MIL}): \mathrm{m}($ oil $)=1: 3$

$\mathrm{T}=50{ }^{\circ} \mathrm{C} ; \mathrm{t}=120 \mathrm{~min} ; \mathrm{n}(\mathrm{O}): \mathrm{n}(\mathrm{S})=6: 1 ; 99.3$

$\mathrm{m}(\mathrm{MIL}): \mathrm{m}($ oil $)=1: 20 ; 6$ recycles

$\mathrm{T}=50^{\circ} \mathrm{C} ; \mathrm{t}=120 \mathrm{~min} ;$ Initial S-content $559.7 \mathrm{ppm} ; \quad 98.5$

$\mathrm{n}(\mathrm{O}): \mathrm{n}(\mathrm{S})=6: 1 ; \mathrm{m}(\mathrm{MIL}): \mathrm{m}(\mathrm{oil})=1: 10$

$\mathrm{T}=60{ }^{\circ} \mathrm{C} ; \mathrm{t}=120 \min ; \mathrm{n}(\mathrm{O}): \mathrm{n}(\mathrm{S})=6: 1 ; \quad 99.9$ $\mathrm{m}(\mathrm{MIL}): \mathrm{m}($ oil $)=1: 5 ; 6$ recycles

$\mathrm{T}=60{ }^{\circ} \mathrm{C} ; \mathrm{t}=120 \mathrm{~min}$; Initial S-content $559.7 \mathrm{ppm} ; \quad 98.8$ $\mathrm{n}(\mathrm{O}): \mathrm{n}(\mathrm{S})=6: 1 ; \mathrm{m}(\mathrm{MIL}): \mathrm{m}(\mathrm{oil})=1: 5$

$\mathrm{T}=50^{\circ} \mathrm{C} ; \mathrm{t}=60 \mathrm{~min}$; Initial S-content $1000 \mathrm{ppm} ; 100$

$\mathrm{n}(\mathrm{O}): \mathrm{n}(\mathrm{S})=6: 1 ; \mathrm{m}(\mathrm{MIL}): \mathrm{m}(\mathrm{oil})=1: 5 ; 6$ recycles

$\mathrm{T}=50^{\circ} \mathrm{C} ; \mathrm{t}=60 \mathrm{~min}$; Initial S-content $559.7 \mathrm{ppm} ; \quad 99.2$ $\mathrm{n}(\mathrm{O}): \mathrm{n}(\mathrm{S})=6: 1 ; \mathrm{m}(\mathrm{MIL}): \mathrm{m}(\mathrm{oil})=3: 10$
76

77

Schiff-base IL:

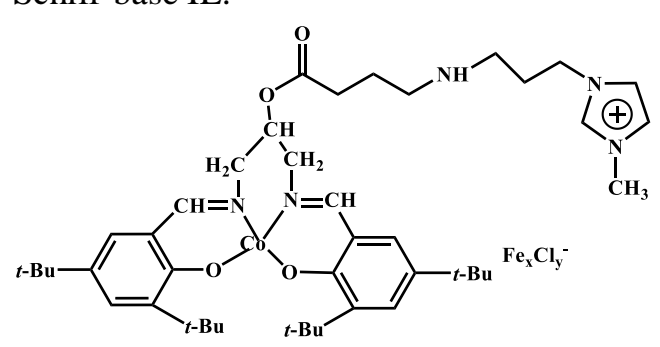


Table S5. Oxidative desulfurization of organic sulfide in metal oxide ILs over the past decade.

\begin{tabular}{|c|c|c|c|c|c|c|}
\hline Catalyst & Extractant & Oxidant & $\begin{array}{l}\text { Oil/Sulfur } \\
\text { compounds }\end{array}$ & Reaction conditions & $\begin{array}{l}\text { S- } \\
\text { removal/\% }\end{array}$ & Ref. \\
\hline \multirow[t]{2}{*}[(\mathrm{C}_{8}\mathrm{H}_{17})_{3}\mathrm{NCH}_{3}]{$_{2} \mathrm{~W}_{6} \mathrm{O}_{19}$} & [Omim]PF6 & $\mathrm{H}_{2} \mathrm{O}_{2}$ & n-octane/DBT & $\begin{array}{l}\mathrm{T}=60^{\circ} \mathrm{C} ; \mathrm{t}=60 \mathrm{~min} ; \text { Initial S-content } 500 \\
\text { ppm; } \mathrm{V}(\mathrm{O})=24 \mu \mathrm{L} ; \mathrm{m}(\mathrm{MIL})=3.5 \mathrm{mg} ; \\
\mathrm{V}(\text { extractant }): \mathrm{V}(\text { oil })=1: 5 ; 15 \text { recycles }\end{array}$ & 97.7 & 41 \\
\hline & & & FCC gasoline & $\begin{array}{l}\mathrm{T}=60^{\circ} \mathrm{C} ; \mathrm{t}=60 \mathrm{~min} ; \text { Initial S-content } 500 \\
\text { ppm; } \mathrm{V}(\mathrm{O})=24 \mu \mathrm{L} ; \mathrm{m}(\mathrm{MIL})=3.5 \mathrm{mg} \\
\mathrm{V}(\text { extractant }): \mathrm{V}(\text { oil })=1: 5\end{array}$ & 74.9 & 41 \\
\hline$\left[\left(\mathrm{CH}_{3}\right) \mathrm{N}\left(\mathrm{n}-\mathrm{C}_{8} \mathrm{H}_{17}\right)_{3}\right]_{2} \mathrm{~W}_{2} \mathrm{O}_{11}$ & {$[\mathrm{Omim}] \mathrm{PF}_{6}$} & $\mathrm{H}_{2} \mathrm{O}_{2}$ & n-octane/DBT & $\begin{array}{l}\mathrm{T}=30^{\circ} \mathrm{C} ; \mathrm{t}=120 \mathrm{~min} ; \text { Initial S-content } 500 \\
\text { ppm; } \mathrm{n}(\mathrm{O}): \mathrm{n}(\mathrm{S})=6: 1 ; \mathrm{V}(\mathrm{MIL}): \mathrm{V}(\text { oil })= \\
1: 50 ; \mathrm{V}(\text { extractant })=1 \mathrm{~mL} ; \text { agitation rate } \\
500 \mathrm{rpm}\end{array}$ & 98.1 & 43 \\
\hline$\left[\left(\mathrm{C}_{6} \mathrm{H}_{13}\right)_{3} \mathrm{PC}_{14} \mathrm{H}_{29}\right]_{2} \mathrm{~W}_{6} \mathrm{O}_{19}$ & - & $\mathrm{H}_{2} \mathrm{O}_{2}$ & n-octane/DBT & $\begin{array}{l}\mathrm{T}=60^{\circ} \mathrm{C} ; \mathrm{t}=60 \mathrm{~min} ; \mathrm{V}(\mathrm{O})=48 \mathrm{~mL} ; \mathrm{V}(\mathrm{oil}) \\
=5 \mathrm{~mL} ; \mathrm{m}(\mathrm{MIL})=0.3705 \mathrm{~g} ; 10 \text { recycles }\end{array}$ & 97.8 & 80 \\
\hline$[\mathrm{PSPy}]_{3} \mathrm{PW}_{12} \mathrm{O}_{40}$ & [Omim]PF6 & $\mathrm{H}_{2} \mathrm{O}_{2}$ & n-octane/DBT & $\begin{array}{l}\mathrm{T}=30^{\circ} \mathrm{C} ; \mathrm{t}=60 \mathrm{~min} ; \text { Initial S-content } 500 \\
\text { ppm; } \mathrm{n}(\mathrm{O}): \mathrm{n}(\mathrm{S}): \mathrm{n}(\mathrm{MIL}) \quad=\quad 480: 120: 1 ; \\
\mathrm{V}(\text { extractant }): \mathrm{V}(\text { oil })=1: 5 ; 9 \text { recycles }\end{array}$ & 99.5 & 81 \\
\hline \multirow[t]{2}{*}[(\mathrm{C}_{18}\mathrm{H}_{37})\mathrm{N}(\mathrm{CH}_{3})_{3}]{$_{7}\left[\mathrm{PW}_{11} \mathrm{O}_{39}\right]$} & [Bmim]PF6 & $\mathrm{H}_{2} \mathrm{O}_{2}$ & n-octane/DBT & $\begin{array}{l}\mathrm{T}=30^{\circ} \mathrm{C} ; \mathrm{t}=60 \mathrm{~min} ; \text { Initial S-content } 500 \\
\text { ppm; } \mathrm{n}(\mathrm{O}): \mathrm{n}(\mathrm{S}): \mathrm{n}(\mathrm{MIL}) \quad=\quad 600: 150: 1 ; \\
\mathrm{V}(\text { extractant }): \mathrm{V}(\text { oil })=1: 5 ; 5 \text { recycles }\end{array}$ & 99.1 & 42 \\
\hline & & & gasoline & $\begin{array}{l}\mathrm{T}=30{ }^{\circ} \mathrm{C} ; \mathrm{t}=180 \mathrm{~min} \text {; Initial S-content } \\
1236 \quad \mathrm{ppm} ; \mathrm{m}(\mathrm{MIL}) \quad=0.0758 \mathrm{~g} \\
\mathrm{~V}(\mathrm{O}): \mathrm{V}(\text { extractant }): \mathrm{V}(\mathrm{oil})=1: 2: 10\end{array}$ & 94.7 & 42 \\
\hline \multirow[t]{2}{*}[\mathrm{MIMPS}]{$_{3} \mathrm{PW}_{12} \mathrm{O}_{40} \cdot 2 \mathrm{H}_{2} \mathrm{O}$} & {$[\mathrm{Omim}] \mathrm{PF}_{6}$} & $\mathrm{H}_{2} \mathrm{O}_{2}$ & n-octane/DBT & $\begin{array}{l}\mathrm{T}=30^{\circ} \mathrm{C} ; \mathrm{t}=60 \mathrm{~min} \text {; Initial S-content } 500 \\
\text { ppm; } \mathrm{n}(\mathrm{O}): \mathrm{n}(\mathrm{S}): \mathrm{n}(\mathrm{MIL}) \quad=\quad 480: 120: 1 ; \\
\mathrm{V}(\text { extractant }): \mathrm{V}(\text { oil })=1: 5 ; 8 \text { recycles }\end{array}$ & 100 & 82 \\
\hline & & & Diesel fuel & $\begin{array}{l}\mathrm{T}=30^{\circ} \mathrm{C} ; \mathrm{t}=180 \mathrm{~min} ; \text { Initial S-content } \\
1113 \mathrm{ppm} ; \mathrm{n}(\mathrm{MIL})=0.65 \mu \mathrm{mol} ; \mathrm{n}(\mathrm{O})= \\
3.12 \mu \mathrm{mol} ; \mathrm{V}(\text { extractant }): \mathrm{V}(\text { oil })=1: 5\end{array}$ & 82.2 & 82 \\
\hline$[\mathrm{Bmim}]_{3} \mathrm{PW}_{12} \mathrm{O}_{40}$ & {$[\mathrm{Omim}] \mathrm{PF}_{6}$} & $\mathrm{H}_{2} \mathrm{O}_{2}$ & n-octane/DBT & $\begin{array}{l}\mathrm{T}=30^{\circ} \mathrm{C} ; \mathrm{t}=60 \mathrm{~min} ; \text { Initial S-content } 500 \\
\text { ppm; } \mathrm{n}(\mathrm{O}): \mathrm{n}(\mathrm{S}): \mathrm{n}(\mathrm{MIL}) \quad=\quad 480: 120: 1 ; \\
\mathrm{V}(\text { extractant }): \mathrm{V}(\mathrm{oil})=1: 5\end{array}$ & 97.9 & 82 \\
\hline$\left[\left(\mathrm{n}-\mathrm{C}_{8} \mathrm{H}_{17}\right)_{3} \mathrm{NCH}_{3}\right]_{3}\left\{\mathrm{PO}_{4}\left[\mathrm{WO}\left(\mathrm{O}_{2}\right)_{2}\right]_{4}\right\}$ & - & $\mathrm{H}_{2} \mathrm{O}_{2}$ & n-octane/DBT & $\begin{array}{l}\mathrm{T}=60^{\circ} \mathrm{C} ; \mathrm{t}=120 \mathrm{~min} ; \mathrm{n}(\mathrm{O}): \mathrm{n}(\mathrm{S})=6: 1 \\
\mathrm{~m}(\text { oil): } \mathrm{m}(\mathrm{MIL})=50 ; \mathrm{V}(\mathrm{oil})=5 \mathrm{~mL} \\
\text { agitation rate } 500 \mathrm{rpm}\end{array}$ & 100 & 80 \\
\hline \multirow[t]{2}{*}[(\mathrm{n}-\mathrm{C}_{12}\mathrm{H}_{25})_{3}\mathrm{NCH}_{3}]{$_{3}\left\{\mathrm{PO}_{4}\left[\mathrm{WO}\left(\mathrm{O}_{2}\right)_{2}\right]_{4}\right\}$} & - & $\mathrm{H}_{2} \mathrm{O}_{2}$ & n-octane/DBT & $\begin{array}{l}\mathrm{T}=60^{\circ} \mathrm{C} ; \mathrm{t}=120 \mathrm{~min} ; \mathrm{n}(\mathrm{O}): \mathrm{n}(\mathrm{S})=6: 1 \\
\mathrm{~m}(\text { oil }): \mathrm{m}(\mathrm{MIL})=50 ; \mathrm{V}(\mathrm{oil})=5 \mathrm{~mL} \\
\text { agitation rate } 500 \mathrm{rpm}\end{array}$ & 100 & 80 \\
\hline & $\mathrm{H}_{2} \mathrm{O}$ & $\mathrm{H}_{2} \mathrm{O}_{2}$ & n-hexane/MPS & $\begin{array}{l}\mathrm{T}=25^{\circ} \mathrm{C} ; \mathrm{t}=2 \mathrm{~min} \text {; Initial S-content } 250 \\
\text { ppm; } \mathrm{n}(\mathrm{O}): \mathrm{n}(\mathrm{S})=1: 1 ; \mathrm{n}(\mathrm{S})=1 \mathrm{mmol} ; \\
\mathrm{m}(\mathrm{MIL})=40 \mathrm{mg} ; \mathrm{V}(\text { extractant })=4 \mathrm{~mL}\end{array}$ & 95 & 83 \\
\hline
\end{tabular}


Table S5. Continued.

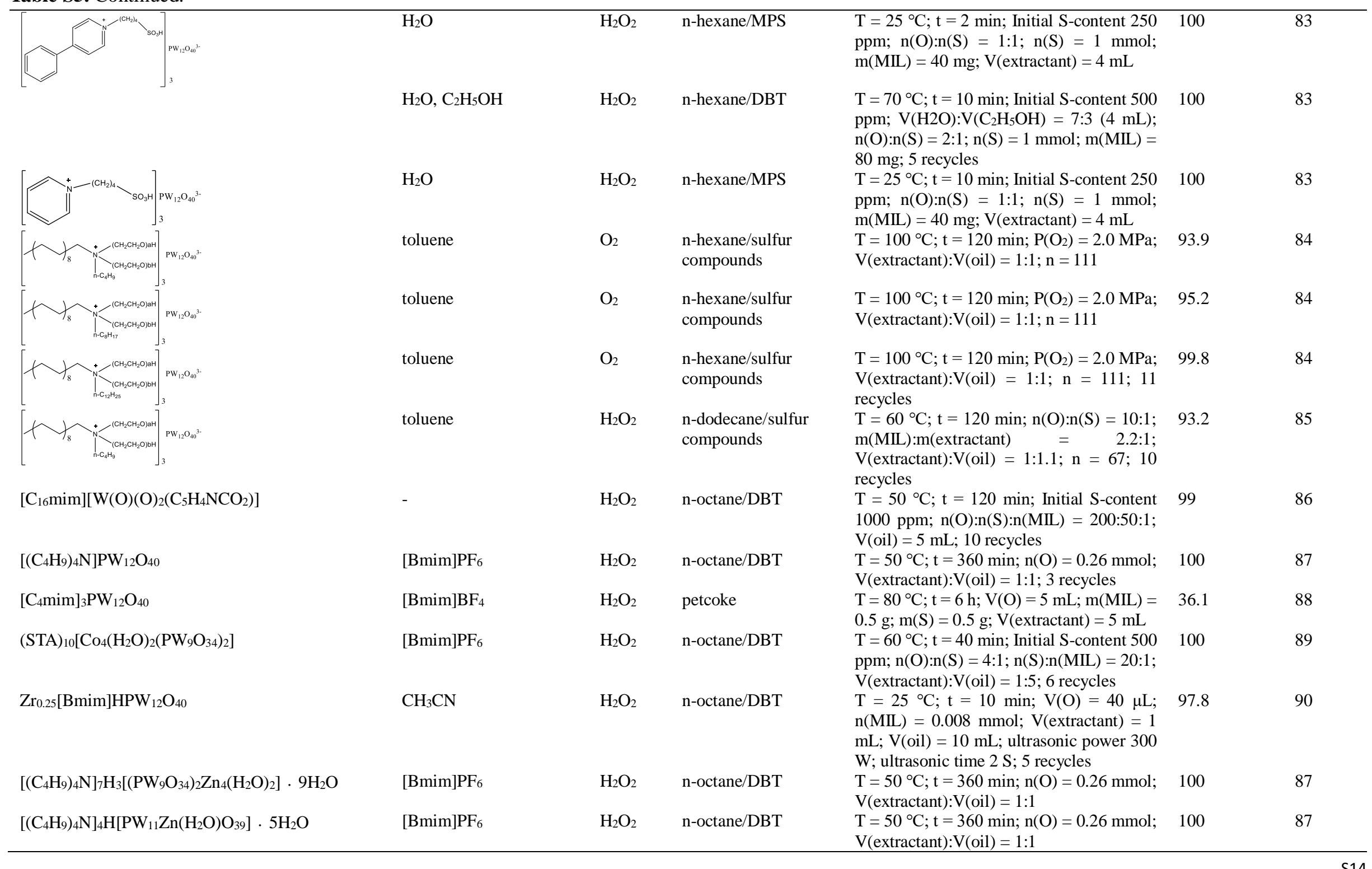


Table S5. Continued.

\begin{tabular}{|c|c|c|c|c|c|c|}
\hline \multirow[t]{3}{*}{$(\mathrm{DODA})_{9} \mathrm{LaW}_{10} \mathrm{O}_{36} \cdot 4 \mathrm{H}_{2} \mathrm{O}$} & \multirow[t]{3}{*}[\mathrm{Omim}]{$\mathrm{PF}_{6}$} & \multirow[t]{3}{*}{$\mathrm{H}_{2} \mathrm{O}_{2}$} & n-octane/DBT & $\mathrm{T}=30^{\circ} \mathrm{C} ; \mathrm{t}=45 \mathrm{~min} ;$ Initial S-content 1000 & 100 & \multirow[t]{3}{*}{91} \\
\hline & & & n-octane/BT & ppm; $\mathrm{n}(\mathrm{O}): \mathrm{n}(\mathrm{S}): \mathrm{n}(\mathrm{MIL})=500: 100: 1 ; \mathrm{V}(\mathrm{O})$ & 65.4 & \\
\hline & & & $\begin{array}{l}\text { n-octane/4,6- } \\
\text { DMDBT }\end{array}$ & $\begin{array}{l}=0.08 \mathrm{~mL} ; \mathrm{V}(\text { extractant })=1 \mathrm{~mL} ; \mathrm{V}(\text { oil })= \\
5 \mathrm{~mL}\end{array}$ & 57.9 & \\
\hline \multirow[t]{3}{*}{$(\mathrm{DDA})_{9} \mathrm{LaW}_{10} \mathrm{O}_{36}$} & \multirow[t]{3}{*}[\mathrm{Omim}]{$\mathrm{PF}_{6}$} & \multirow[t]{3}{*}{$\mathrm{H}_{2} \mathrm{O}_{2}$} & n-octane/BT & $\mathrm{T}=30^{\circ} \mathrm{C} ; \mathrm{t}=45 \mathrm{~min} ;$ Initial S-content 1000 & 90.2 & \multirow[t]{3}{*}{91} \\
\hline & & & n-octane/4,6- & ppm; $\mathrm{n}(\mathrm{O}): \mathrm{n}(\mathrm{S}): \mathrm{n}(\mathrm{MIL})=500: 100: 1 ; \mathrm{V}(\mathrm{O})$ & 86.9 & \\
\hline & & & $\begin{array}{l}\text { DMDBT } \\
\text { n-octane/DBT }\end{array}$ & $\begin{array}{l}=0.08 \mathrm{~mL} ; \mathrm{V}(\text { extractant })=1 \mathrm{~mL} ; \mathrm{V}(\text { oil })= \\
5 \mathrm{~mL}\end{array}$ & 100 & \\
\hline \multirow[t]{2}{*}{$(\mathrm{TSA})_{9} \mathrm{LaW}_{10} \mathrm{O}_{36}$} & \multirow[t]{2}{*}[\mathrm{Omim}]{$\mathrm{PF}_{6}$} & \multirow[t]{2}{*}{$\mathrm{H}_{2} \mathrm{O}_{2}$} & n-octane/BT & $\mathrm{T}=30^{\circ} \mathrm{C} ; \mathrm{t}=45 \mathrm{~min} ;$ Initial S-content 1000 & 78.5 & \multirow[t]{2}{*}{91} \\
\hline & & & $\begin{array}{l}\text { n-octane/4,6- } \\
\text { DMDBT }\end{array}$ & $\begin{array}{l}\text { ppm; } \mathrm{n}(\mathrm{O}): \mathrm{n}(\mathrm{S}): \mathrm{n}(\mathrm{MIL})=500: 100: 1 ; \mathrm{V}(\mathrm{O}) \\
=0.08 \mathrm{~mL} ; \mathrm{V}(\text { extractant })=1 \mathrm{~mL} ; \mathrm{V}(\mathrm{oil})= \\
5 \mathrm{~mL}\end{array}$ & 70.4 & \\
\hline$\left[\left(\mathrm{CH}_{3}\right) \mathrm{N}\left(\mathrm{n}-\mathrm{C}_{8} \mathrm{H}_{17}\right)_{3}\right]_{2} \mathrm{Mo}_{2} \mathrm{O}_{11}$ & - & $\mathrm{H}_{2} \mathrm{O}_{2}$ & n-octane/DBT & $\begin{array}{l}\mathrm{T}=30^{\circ} \mathrm{C} ; \mathrm{t}=120 \mathrm{~min} ; \text { Initial S-content } 500 \\
\text { ppm; } \mathrm{n}(\mathrm{O}): \mathrm{n}(\mathrm{S})=8: 1 ; \mathrm{V}(\mathrm{MIL}): \mathrm{V}(\text { oil })= \\
1: 40 ; 10 \text { recycles }\end{array}$ & 96.2 & 43 \\
\hline$\left[\left(\mathrm{C}_{12} \mathrm{H}_{25}\right) \mathrm{N}\left(\mathrm{CH}_{3}\right)_{3}\right] \mathrm{Mo}_{8} \mathrm{O}_{26}$ & {$\left[\left(\mathrm{CH}_{2}\right)_{3} \mathrm{SO}_{3} \mathrm{HMIm}_{\mathrm{B}}\right] \mathrm{BF}_{4}$} & $\mathrm{H}_{2} \mathrm{O}_{2}$ & n-octane/DBT & $\begin{array}{l}\mathrm{T}=70^{\circ} \mathrm{C} ; \mathrm{t}=180 \mathrm{~min} ; \mathrm{n}(\mathrm{O}): \mathrm{n}(\mathrm{S})=4: 1 \\
\mathrm{n}(\mathrm{S}): \mathrm{n}(\mathrm{MIL})=100 ; \mathrm{V}(\text { oil })=5 \mathrm{~mL} \\
\mathrm{~V}(\text { extractant })=1 \mathrm{~mL} ; 6 \text { recycles }\end{array}$ & 98.3 & 92 \\
\hline$\left[\left(\mathrm{C}_{16} \mathrm{H}_{33}\right) \mathrm{N}\left(\mathrm{CH}_{3}\right)_{3}\right] \mathrm{Mog}_{8} \mathrm{O}_{26}$ & - & $\mathrm{H}_{2} \mathrm{O}_{2}$ & n-octane/DBT & $\begin{array}{l}\mathrm{T}=70^{\circ} \mathrm{C} ; \mathrm{t}=180 \mathrm{~min} ; \mathrm{n}(\mathrm{O}): \mathrm{n}(\mathrm{S})=4: 1 \\
\mathrm{n}(\mathrm{S}): \mathrm{n}(\mathrm{MIL})=100 ; \quad \mathrm{V}(\mathrm{oil})=5 \mathrm{~mL} \\
\mathrm{~V}(\text { extractant })=1 \mathrm{~mL}\end{array}$ & 99 & 92 \\
\hline$\left[\left(\mathrm{C}_{4} \mathrm{H}_{9}\right)_{4} \mathrm{~N}\right]_{6} \mathrm{Mo}_{7} \mathrm{O}_{24}$ & {$[\mathrm{Bmim}] \mathrm{BF}_{4}$} & $\mathrm{H}_{2} \mathrm{O}_{2}$ & n-octane/DBT & $\begin{array}{l}\mathrm{T}=50^{\circ} \mathrm{C} ; \mathrm{t}=180 \mathrm{~min} ; \text { Initial S-content } 500 \\
\text { ppm; } \mathrm{n}(\mathrm{O}): \mathrm{n}(\mathrm{S})=5: 1 ; \mathrm{V}(\text { extractant }): \mathrm{V}(\text { oil }) \\
=1: 5 ; 6 \text { recycles }\end{array}$ & 99 & 93 \\
\hline$\left(\mathrm{NH}_{4}\right)_{6} \mathrm{Mo}_{7} \mathrm{O}_{24} \cdot 4 \mathrm{H}_{2} \mathrm{O}$ & {$[\mathrm{Bmim}] \mathrm{DBP}$} & $\mathrm{H}_{2} \mathrm{O}_{2}$ & $\begin{array}{l}\text { n-octane/4,6- } \\
\text { DMDBT }\end{array}$ & $\begin{array}{l}\mathrm{T}=50^{\circ} \mathrm{C} ; \mathrm{t}=180 \mathrm{~min} ; \mathrm{m}(\mathrm{O})=0.2 \mathrm{~g} ; \\
\mathrm{m}(\mathrm{MIL})=0.1 \mathrm{~g} ; \mathrm{m}(\text { extractant })=1 \mathrm{~g} ; \mathrm{m}(\text { oil }) \\
=5 \mathrm{~g} ; 6 \text { recycles }\end{array}$ & 89.2 & 94 \\
\hline$\left(\mathrm{NH}_{4}\right)_{2}(\mathrm{MimAm})_{40}\left[\mathrm{Mo}_{132} \mathrm{O}_{372}\left(\mathrm{CH}_{3} \mathrm{COO}\right)_{30}\left(\mathrm{H}_{2} \mathrm{O}\right)_{72}\right]$ & $\mathrm{H}_{2} \mathrm{O}$ & $\mathrm{H}_{2} \mathrm{O}_{2}$ & thioanisole & $\begin{array}{l}\mathrm{T}=25^{\circ} \mathrm{C} ; \mathrm{t}=10 \mathrm{~min} ; \mathrm{n}(\mathrm{O})=2 \mathrm{mmol} \\
\mathrm{m}(\mathrm{MIL})=200 \mathrm{mg} ; \mathrm{V}(\text { extractant })=3 \mathrm{~mL} \\
\mathrm{n}(\mathrm{S})=1 \mathrm{mmol} ; 7 \text { recycles }\end{array}$ & 100 & 95 \\
\hline \multirow[t]{3}{*}[\mathrm{PyPS}]{$_{3}\left(\mathrm{NH}_{4}\right)_{3} \mathrm{Mo}_{7} \mathrm{O}_{24}$} & {$[\mathrm{Omim}] \mathrm{BF}_{4}$} & $\mathrm{H}_{2} \mathrm{O}_{2}$ & n-octane/DBT & $\begin{array}{l}\mathrm{T}=25^{\circ} \mathrm{C} ; \mathrm{t}=60 \mathrm{~min} ; \mathrm{n}(\mathrm{O}): \mathrm{n}(\mathrm{S})=3: 1 ; \\
\mathrm{n}(\mathrm{MIL}) \quad=\quad 0.0056 \quad \mathrm{mmol} ; \\
\mathrm{V}(\text { extractant }): \mathrm{V}(\text { oil })=1: 10 ; 5 \text { recycles }\end{array}$ & 99 & 38 \\
\hline & & & Commercial diesel & $\begin{array}{l}\mathrm{T}=25^{\circ} \mathrm{C} ; \mathrm{t}=60 \mathrm{~min} ; \text { Initial S-content } 242 \\
\text { ppm; } \mathrm{n}(\mathrm{O}): \mathrm{n}(\mathrm{S})=3: 1 ; \mathrm{n}(\mathrm{MIL})=0.0056 \\
\text { mmol; } \mathrm{V}(\text { extractant }): \mathrm{V}(\mathrm{oil})=1: 1\end{array}$ & 96 & \\
\hline & - & $\mathrm{H}_{2} \mathrm{O}_{2}$ & Dodecane/MeSPh & $\begin{array}{l}\mathrm{T}=60^{\circ} \mathrm{C} ; \mathrm{t}=120 \mathrm{~min} ; \text { Initial S-content } \\
2000 \mathrm{ppm} ; \mathrm{n}(\mathrm{O}): \mathrm{n}(\mathrm{S})=6: 1 ; \mathrm{n}(\mathrm{S}): \mathrm{n}(\mathrm{MIL})= \\
24: 1 ; \mathrm{V}(\mathrm{oil})=5 \mathrm{~mL}\end{array}$ & 86 & 96 \\
\hline
\end{tabular}


Table S5. Continued.

\begin{tabular}{|c|c|c|c|c|c|c|}
\hline \multirow{4}{*}[\mathrm{Hmim}]{$_{5} \mathrm{PMo}_{10} \mathrm{~V}_{2} \mathrm{O}_{40}$} & \multirow{4}{*}[\mathrm{Hmim}]{$\mathrm{BF}_{4}$} & \multirow{4}{*}{$\mathrm{H}_{2} \mathrm{O}_{2}$} & \multirow[t]{2}{*}{ gasoline } & \multirow{4}{*}{$\begin{array}{l}\mathrm{T}=30^{\circ} \mathrm{C} ; \mathrm{t}=180 \mathrm{~min} ; \text { Initial S-content } \\
1236 \mu \mathrm{g} / \mathrm{g} ; \mathrm{m}(\mathrm{MIL})=0.07 \mathrm{~g} ; \mathrm{V}(\mathrm{O})=0.5 \\
\mathrm{~mL} ; \mathrm{V}(\text { extractant })=1 \mathrm{~mL} ; \mathrm{V}(\text { oil })=5 \mathrm{~mL} \\
\mathrm{~T}=60^{\circ} \mathrm{C} ; \mathrm{t}=100 \mathrm{~min} ; \text { Initial S-content } 500 \\
\text { ppm; } \mathrm{n}(\mathrm{O}): \mathrm{n}(\mathrm{S})=4: 1 ; \mathrm{n}(\mathrm{MIL}): \mathrm{n}(\mathrm{S})= \\
0.062: 1 ; \mathrm{V}(\text { extractant }): \mathrm{V}(\text { oil })=1: 5 ; 6 \\
\text { recycles }\end{array}$} & \multirow[t]{2}{*}{92.1} & \multirow[t]{2}{*}{98} \\
\hline & & & & & & \\
\hline & & & n-octane/DBT & & 99.1 & 99 \\
\hline & & & & & & \\
\hline \multirow[t]{3}{*}[\mathrm{Bmim}]{$_{5}\left[\mathrm{PMo}{ }_{11} \mathrm{Co}\left(\mathrm{H}_{2} \mathrm{O}\right) \mathrm{O}_{39}\right]$} & \multirow[t]{3}{*}[\mathrm{Omim}]{$\mathrm{BF}_{4}$} & \multirow[t]{3}{*}{$\mathrm{H}_{2} \mathrm{O}_{2}$} & n-octane/DBT & $\begin{array}{l}\mathrm{T}=50^{\circ} \mathrm{C} ; \mathrm{t}=80 \min ; \mathrm{n}(\mathrm{O}): \mathrm{n}(\mathrm{S})=5: 1 \\
\mathrm{n}(\mathrm{MIL})=4 \mu \mathrm{mol} ; \mathrm{V}(\text { extractant })=1 \mathrm{~mL} \\
\mathrm{~V}(\text { oil })=5 \mathrm{~mL} ; 8 \text { recycles }\end{array}$ & 99.2 & \multirow[t]{3}{*}{39} \\
\hline & & & n-octane/BT & $\begin{array}{l}\mathrm{T}=50^{\circ} \mathrm{C} ; \mathrm{t}=80 \min ; \mathrm{n}(\mathrm{O}): \mathrm{n}(\mathrm{S})=5: 1 \\
\mathrm{n}(\mathrm{MIL})=4 \mu \mathrm{mol} ; \mathrm{V}(\text { extractant })=1 \mathrm{~mL} \\
\mathrm{~V}(\text { oil })=5 \mathrm{~mL}\end{array}$ & 65 & \\
\hline & & & $\begin{array}{l}\text { n-octane/4,6- } \\
\text { DMDBT }\end{array}$ & $\begin{array}{l}\mathrm{T}=50^{\circ} \mathrm{C} ; \mathrm{t}=80 \mathrm{~min} ; \mathrm{n}(\mathrm{O}): \mathrm{n}(\mathrm{S})=5: 1 \\
\mathrm{n}(\mathrm{MIL})=4 \mu \mathrm{mol} ; \mathrm{V}(\text { extractant })=1 \mathrm{~mL} \\
\mathrm{~V}(\text { oil })=5 \mathrm{~mL}\end{array}$ & 87 & \\
\hline$\left(\mathrm{C}_{4} \mathrm{H}_{9} \mathrm{~N}\right)_{4}\left[\mathrm{NiMo}_{6} \mathrm{O}_{24} \mathrm{H}_{6}\right]$ & {$\left[\mathrm{Bmim}_{\mathrm{PF}} \mathrm{PF}_{6}\right.$} & $\mathrm{H}_{2} \mathrm{O}_{2}$ & n-octane/DBT & $\begin{array}{l}\mathrm{T}=30^{\circ} \mathrm{C} ; \mathrm{t}=180 \mathrm{~min} ; \text { Initial S-content } 500 \\
\text { ppm; } \mathrm{n}(\mathrm{O}): \mathrm{n}(\mathrm{S})=5: 1 ; \mathrm{n}(\mathrm{Ni}): \mathrm{n}(\mathrm{S})=1: 100 ; \\
\mathrm{V}(\text { extractant }): \mathrm{V}(\text { oil })=1: 5 ; 6 \text { recycles }\end{array}$ & 98 & 100 \\
\hline$\left(\mathrm{C}_{4} \mathrm{H}_{9} \mathrm{~N}\right)_{4}\left[\mathrm{NiMo}_{6} \mathrm{O}_{24} \mathrm{H}_{6}\right]$ & {$\left[\mathrm{Bmim}_{\mathrm{P}} \mathrm{PF}_{6}\right.$} & $\mathrm{H}_{2} \mathrm{O}_{2}$ & Commercial diesel & $\begin{array}{l}\mathrm{T}=60^{\circ} \mathrm{C} ; \mathrm{t}=240 \mathrm{~min} ; \text { Initial } \mathrm{S} \text {-content } 700 \\
\mathrm{ppm} ; \mathrm{V}(\mathrm{O})=0.4 \mathrm{~mL} ; \mathrm{n}(\mathrm{Ni}): \mathrm{n}(\mathrm{S})=1: 20 ; \\
\mathrm{V}(\text { extractant })=1 \mathrm{~mL} ; \mathrm{V}(\mathrm{oil})=5 \mathrm{~mL} ; 6 \\
\text { recycles }\end{array}$ & 96.3 & 100 \\
\hline$(\mathrm{DODMAC})_{3} \mathrm{Co}(\mathrm{OH})_{6} \mathrm{Mo}_{6} \mathrm{O}_{18} \cdot 3 \mathrm{H}_{2} \mathrm{O}$ & {$[\mathrm{Opy}] \mathrm{BF}_{4}$} & $\mathrm{O}_{2}$ & decalin /DBT & $\begin{array}{l}\mathrm{T}=90^{\circ} \mathrm{C} ; \mathrm{t}=8 \mathrm{~h} ; \text { Initial S-content } 500 \\
\mu \mathrm{g} / \mathrm{g} ; \mathrm{m}(\mathrm{MIL})=10 \mathrm{mg} ; \mathrm{V} \text { (extractant })=4 \\
\mathrm{~mL} ; \mathrm{V}(\text { oil })=20 \mathrm{~mL} ; \mathrm{O}_{2} \text { flow rate } 60 \\
\mathrm{~mL} / \mathrm{min} ; 8 \text { recycles }\end{array}$ & 100 & 101 \\
\hline$\left[\mathrm{C}_{4} \mathrm{mim}\right]\left[\mathrm{ReO}_{4}\right]$ & {$\left[\mathrm{Bmim}_{\mathrm{B}} \mathrm{BF}_{4}\right.$} & $\mathrm{H}_{2} \mathrm{O}_{2}$ & thioanisole & $\begin{array}{l}\mathrm{T}=60^{\circ} \mathrm{C} ; \mathrm{t}=90 \mathrm{~min} ; \text { Initial S-content } 1000 \\
\text { ppm; } \mathrm{n}(\mathrm{S})=10 \mathrm{mmol} ; \mathrm{MIL}=5 \mathrm{~mol} \% ; \mathrm{n}(\mathrm{O}) \\
=40 \mathrm{mmol} ; \mathrm{V}(\text { extractant })=2 \mathrm{~mL} ; 10 \\
\text { recycles }\end{array}$ & 96 & 102 \\
\hline $\int_{2} \mathrm{vo}_{3}^{2-}$ & - & $\mathrm{H}_{2} \mathrm{O}_{2}$ & Dodecane/MeSPh & $\begin{array}{l}\mathrm{T}=40^{\circ} \mathrm{C} ; \mathrm{t}=120 \mathrm{~min} ; \text { Initial S-content } \\
2000 \mathrm{ppm} ; \mathrm{n}(\mathrm{O}): \mathrm{n}(\mathrm{S})=6: 1 ; \mathrm{n}(\mathrm{S}): \mathrm{n}(\mathrm{MIL})= \\
24: 1 ; \mathrm{V}(\mathrm{oil})=5 \mathrm{~mL}\end{array}$ & 10 & 37 \\
\hline
\end{tabular}


Table S6. Oxidation desulfurization of organic sulfide in metal complex ILs over the past decade.

\begin{tabular}{|c|c|c|c|c|c|c|}
\hline Catalyst & Extractant & Oxidant & Oil/Sulfur compounds & Reaction conditions & S-removal/\% & Ref. \\
\hline (Porp)Fe(III)Cl & {$[\mathrm{Bmim}] \mathrm{PF}_{6}$} & $\mathrm{H}_{2} \mathrm{O}_{2}$ & n-octane/DBT & $\begin{array}{l}\mathrm{T}=60{ }^{\circ} \mathrm{C} ; \mathrm{t}=4 \mathrm{~h} ; \mathrm{n}(\mathrm{O}): \mathrm{n}(\mathrm{S})=4: 1 ; \mathrm{n}(\mathrm{MIL}): \mathrm{n}(\mathrm{S})=1: 100 \\
\mathrm{~V}(\text { extractant })=2 \mathrm{~mL} ; \mathrm{V}(\text { oil })=5 \mathrm{~mL}\end{array}$ & 100 & 103 \\
\hline \multirow{3}{*}{$\mathrm{Fe}^{\mathrm{III}} \mathrm{TPP}\left(\mathrm{PF}_{6}\right)$} & \multirow{3}{*}[\mathrm{Bmim}]{$\mathrm{PF}_{6}$} & \multirow{3}{*}{$\mathrm{H}_{2} \mathrm{O}_{2}$} & Diesel fuel & $\begin{array}{l}\mathrm{T}=60^{\circ} \mathrm{C} ; \mathrm{t}=4 \mathrm{~h} ; \mathrm{n}(\mathrm{O}): \mathrm{n}(\mathrm{S})=6: 1 ; \mathrm{n}(\mathrm{MIL}): \mathrm{n}(\mathrm{S})=1: 100 \\
\mathrm{~V}(\text { extractant })=2 \mathrm{~mL} ; \mathrm{V}(\text { oil })=5 \mathrm{~mL}\end{array}$ & 48.2 & \\
\hline & & & n-octane/DBT & $\begin{array}{l}\mathrm{T}=60{ }^{\circ} \mathrm{C} ; \mathrm{t}=4 \mathrm{~h} ; \mathrm{n}(\mathrm{O}): \mathrm{n}(\mathrm{S})=3: 1 ; \mathrm{n}(\mathrm{MIL}): \mathrm{n}(\mathrm{S})=1: 100 \\
\mathrm{~V}(\text { extractant })=2 \mathrm{~mL} ; \mathrm{V}(\text { oil })=5 \mathrm{~mL}\end{array}$ & 99.5 & 104 \\
\hline & & & Diesel fuel & $\begin{array}{l}\mathrm{T}=60{ }^{\circ} \mathrm{C} ; \mathrm{t}=4 \mathrm{~h} ; \mathrm{n}(\mathrm{O}): \mathrm{n}(\mathrm{S})=5: 1 ; \mathrm{n}(\mathrm{MIL}): \mathrm{n}(\mathrm{S})=1: 100 \\
\mathrm{~V}(\text { extractant })=2 \mathrm{~mL} ; \mathrm{V}(\text { oil })=5 \mathrm{~mL}\end{array}$ & 59.3 & \\
\hline $\mathrm{Fe}^{\mathrm{III}} \mathrm{TPP}\left(\mathrm{BF}_{4}\right)$ & {$[\mathrm{Bmim}] \mathrm{PF}_{6}$} & $\mathrm{H}_{2} \mathrm{O}_{2}$ & n-octane/DBT & $\begin{array}{l}\mathrm{T}=60{ }^{\circ} \mathrm{C} ; \mathrm{t}=4 \mathrm{~h} ; \mathrm{n}(\mathrm{O}): \mathrm{n}(\mathrm{S})=3: 1 ; \mathrm{n}(\mathrm{MIL}): \mathrm{n}(\mathrm{S})=1: 100 \\
\mathrm{~V}(\text { extractant })=2 \mathrm{~mL} ; \mathrm{V}(\mathrm{oil})=5 \mathrm{~mL}\end{array}$ & 98.8 & 104 \\
\hline $\mathrm{Fe}^{\mathrm{III}} \mathrm{TPPCl}$ & {$[\mathrm{Bmim}] \mathrm{PF}_{6}$} & $\mathrm{H}_{2} \mathrm{O}_{2}$ & n-octane/DBT & $\begin{array}{l}\mathrm{T}=60{ }^{\circ} \mathrm{C} ; \mathrm{t}=4 \mathrm{~h} ; \mathrm{n}(\mathrm{O}): \mathrm{n}(\mathrm{S})=3: 1 ; \mathrm{n}(\mathrm{MIL}): \mathrm{n}(\mathrm{S})=1: 100 \\
\mathrm{~V}(\text { extractant })=2 \mathrm{~mL} ; \mathrm{V}(\text { oil })=5 \mathrm{~mL}\end{array}$ & 93.2 & 104 \\
\hline
\end{tabular}


Table S7. Oxidation desulfurization of $\mathrm{H}_{2} \mathrm{~S}$ in MILs over the past decade.

\begin{tabular}{|c|c|c|c|c|c|c|c|c|c|c|}
\hline \multirow[b]{2}{*}{ MIL } & \multirow[b]{2}{*}{ Solvent } & \multicolumn{3}{|c|}{$\mathbf{H}_{2} \mathrm{~S}$} & \multirow[b]{2}{*}{$\mathbf{T} /{ }^{\circ} \mathbf{C}$} & \multirow[b]{2}{*}{$\mathrm{t} / \mathrm{min}$} & \multirow{2}{*}{$\begin{array}{l}\text { Removal } \\
\text { efficiency/ } \\
\%\end{array}$} & \multirow{2}{*}{$\begin{array}{l}\text { Sulfur } \\
\text { capacity }\end{array}$} & \multirow[b]{2}{*}{ Regeneration conditions } & \multirow[b]{2}{*}{ Ref. } \\
\hline & & $\mathrm{C}_{0}$ & P/MPa & $\begin{array}{l}\mathrm{F} / \mathrm{mL} \cdot \mathbf{m i n} \\
-1\end{array}$ & & & & & & \\
\hline$\left[\mathrm{C}_{4} \mathrm{mim}\right] \mathrm{Cl} / 2 \mathrm{FeCl}_{3}$ & - & $\begin{array}{l}1428.6- \\
285714 \mathrm{ppm}\end{array}$ & - & $30-60$ & $30-90$ & 30 & $\geq 99$ & $0.31 \mathrm{~g} / \mathrm{L}$ & $\mathrm{O}_{2}$ flow rate $10-70 \mathrm{~mL} / \mathrm{min}$ & 105 \\
\hline$\left[\mathrm{C}_{4} \mathrm{mim}\right] \mathrm{Cl} / 2 \mathrm{FeCl}_{3}$ & - & $1 \%$ & - & 80 & 105 & 70 & 100 & $0.87 \mathrm{~g} / \mathrm{L}$ & $\begin{array}{l}\mathrm{T}=25^{\circ} \mathrm{C} ; \text { air bubbling for } 80 \mathrm{~h} \\
\mathrm{~T}=50^{\circ} \mathrm{C} ; \text { air bubbling for } 20 \mathrm{~h} \\
\mathrm{~T}=25^{\circ} \mathrm{C} ; \mathrm{O}_{2} \text { bubbling for } 7 \mathrm{~h} \\
\mathrm{~T}=50^{\circ} \mathrm{C} ; \mathrm{O}_{2} \text { bubbling for } 3 \mathrm{~h}\end{array}$ & 106 \\
\hline$\left[\mathrm{C}_{4} \mathrm{mim}\right] \mathrm{Cl} / 2 \mathrm{FeCl}_{3}$ & - & $99.9 \%$ & 0.05 & 15 & RT & 20 & - & $1.984 \%$ & $\begin{array}{l}\mathrm{T}=\mathrm{RT} ; \mathrm{O}_{2} \text { bubbling for } 30-50 \mathrm{~min} ; 3 \\
\text { recycles }\end{array}$ & 107 \\
\hline$\left[\mathrm{C}_{4} \mathrm{mim}\right] \mathrm{Cl} / 2 \mathrm{FeCl}_{3}$ & - & $3.5 \mathrm{~g} / \mathrm{m}^{3}$ & $0.015-0.02$ & $6.7 \times 10^{5}$ & 40 & - & 86.8 & $0.1-0.3 \mathrm{~kg} / \mathrm{m}^{3}$ & $\begin{array}{l}\mathrm{T}=50-80{ }^{\circ} \mathrm{C} ; \mathrm{L}(\mathrm{MIL})=0.17 \mathrm{~m} 3 / \mathrm{h} \\
\mathrm{V}(\text { air })=0.062 \mathrm{~m} 3 / \mathrm{h} ; 8-10 \mathrm{~min}\end{array}$ & 108 \\
\hline$\left[\mathrm{C}_{4} \mathrm{mim}\right] \mathrm{Cl} / 2 \mathrm{FeCl}_{3}$ & - & $99.9 \%$ & 0.05 & 15 & 60 & 20 & - & - & - & 109 \\
\hline $2\left[\mathrm{C}_{4} \mathrm{mim}\right] \mathrm{Cl} / \mathrm{FeCl}_{3}$ & - & $99.9 \%$ & 0.05 & 15 & 60 & 20 & - & - & - & 109 \\
\hline \multirow[t]{3}{*}[\mathrm{C}_{4}\mathrm{mim}]{$\mathrm{Cl} / 2 \mathrm{FeCl}_{3}$} & - & $5 \%$ & - & $60-80$ & RT & 15 & - & $0.38 \mathrm{~g} / \mathrm{L}$ & $\begin{array}{l}\mathrm{N}_{2} \text { blow for } 30 \mathrm{~min} ; \mathrm{O}_{2} \text { bubbling for } \\
30 \mathrm{~min}\end{array}$ & 110 \\
\hline & - & $20 \%$ & - & $60-80$ & RT & 15 & - & $2.62 \mathrm{~g} / \mathrm{L}$ & $\begin{array}{l}\mathrm{N}_{2} \text { blow for } 30 \mathrm{~min} ; \mathrm{O}_{2} \text { bubbling for } \\
30 \mathrm{~min}\end{array}$ & \\
\hline & - & $99.9 \%$ & - & $60-80$ & RT & 15 & - & $14.48 \mathrm{~g} / \mathrm{L}$ & $\begin{array}{l}\mathrm{N}_{2} \text { blow for } 30 \mathrm{~min} ; \mathrm{O}_{2} \text { bubbling for } \\
30 \mathrm{~min}\end{array}$ & \\
\hline$\left[\mathrm{C}_{4} \mathrm{mim}\right] \mathrm{Cl} / 2 \mathrm{FeCl}_{3}$ & $\mathrm{H}_{2} \mathrm{O}$ & $99.9 \%$ & 0.05 & 15 & 60 & 20 & $1.15 \%$ & - & $\begin{array}{l}\mathrm{O}_{2} \text { flow rate } 30 \mathrm{~mL} / \mathrm{min} ; \mathrm{O}_{2} \text { pressure } \\
0.05 \mathrm{MPa} ; 40 \mathrm{~min}\end{array}$ & 111 \\
\hline$\left[\mathrm{C}_{4} \mathrm{mim}\right] \mathrm{Cl} / 0.3 \mathrm{FeCl}_{3}$ & - & $99.9 \%$ & - & 15 & 80 & $\begin{array}{l}276- \\
360\end{array}$ & - & $7.925 \mathrm{~g} /(\mathrm{h} \cdot \mathrm{L})$ & $\mathrm{O}_{2}$ flow rate $30 \mathrm{~mL} / \mathrm{min} ; 4.6-6 \mathrm{~h}$ & 112 \\
\hline
\end{tabular}


Table S7. Continued.

\begin{tabular}{|c|c|c|c|c|c|c|c|c|c|c|}
\hline $\begin{array}{l}{\left[\mathrm{C}_{4} \mathrm{mim}\right] \mathrm{Cl} / 0.5 \mathrm{FeCl}_{3} /} \\
0.7 \mathrm{FeCl}_{2}\end{array}$ & DMF & $1 \%$ & - & 30 & 30 & 300 & 100 & - & $\begin{array}{l}\text { Work electrode: } \mathrm{Pt} \text {; counter electrode: } \\
\mathrm{Pt} \text {; reference electrode: } \mathrm{Ag} / \mathrm{AgCl} \text {; } \\
+0.8 \mathrm{~V} ; 2.5 \mathrm{~h} ; 6 \text { recycles }\end{array}$ & 113 \\
\hline $\begin{array}{l}{\left[\mathrm{C}_{4} \mathrm{mim}\right] \mathrm{Cl} / 2 \mathrm{FeCl}_{3} / 1} \\
5 \mathrm{ZnCl}_{12}\end{array}$ & DMI & $10000 \mathrm{ppm}$ & - & 30 & 25 & 100 & $>90$ & - & - & 114 \\
\hline $1.6 \mathrm{Et}_{3} \mathrm{NHCl} / \mathrm{FeCl}_{3}$ & - & $832 \mathrm{mg} / \mathrm{m}^{3}$ & - & $<400$ & $80-180$ & 180 & 100 & $6.36 \mathrm{~g} / \mathrm{L}$ & $\begin{array}{l}\mathrm{T}=120^{\circ} \mathrm{C} \text {; air flow rate } 300 \mathrm{~mL} / \mathrm{min} \text {; } \\
120 \mathrm{~min}\end{array}$ & 22 \\
\hline $1.5 \mathrm{Et}_{3} \mathrm{NHCl} / \mathrm{FeCl}_{3}$ & - & $>99.5 \%$ & - & 10 & 30 & 120 & 87.9 & $2.178 \mathrm{wt} \%$ & $\begin{array}{l}\mathrm{T}=100^{\circ} \mathrm{C} \text {; air bubbling; } 10.1 \mathrm{kPa} ; 50 \\
\text { min; } 5 \text { recycles }\end{array}$ & 115 \\
\hline$[\mathrm{A} 336] \mathrm{Cl} / \mathrm{FeCl}_{3}$ & {$[\mathrm{Bmim}] \mathrm{OH}$} & $99.9 \%$ & 0.05 & 15 & RT & - & $2.13 \%$ & - & - & 116 \\
\hline$\left[\mathrm{C}_{4} \mathrm{mim}\right] \mathrm{PMo}_{12}$ & {$[\mathrm{Bmim}] \mathrm{Cl}$} & $1300 \mathrm{mg} / \mathrm{m}^{3}$ & 0.1 & 100 & 200 & 180 & 95 & - & $\begin{array}{l}\mathrm{T}=200^{\circ} \mathrm{C} \text {; air flow rate } 300 \mathrm{~mL} / \mathrm{min} ; \\
5 \mathrm{~h} ; 3 \text { recycles }\end{array}$ & 50 \\
\hline$\left[\mathrm{C}_{4} \mathrm{mim}\right] \mathrm{PMo}_{10} \mathrm{~V}_{2}$ & {$[\mathrm{Bmim}] \mathrm{Cl}$} & $2000 \mathrm{mg} / \mathrm{m}^{3}$ & 0.1 & 100 & 200 & 360 & 90 & - & $\begin{array}{l}\mathrm{T}=200^{\circ} \mathrm{C} \text {; air flow rate } 300 \mathrm{~mL} / \mathrm{min} ; \\
5 \mathrm{~h} ; 3 \text { recycles }\end{array}$ & 50 \\
\hline $\begin{array}{l}{\left[\mathrm{CH}_{3}\left(\mathrm{CH}_{2}\right)_{15} \mathrm{~N}\left(\mathrm{CH}_{3}\right)_{3}\right.} \\
]_{3}\left\{\mathrm{PO}_{4}\left[\mathrm{MoO}\left(\mathrm{O}_{2}\right)_{2}\right]_{4}\right\}\end{array}$ & {$[\mathrm{Bmim}] \mathrm{HCO}_{3}$} & $690 \mathrm{mg} / \mathrm{m}^{3}$ & - & 200 & 95 & 120 & 100 & - & $\begin{array}{l}\mathrm{T}=95{ }^{\circ} \mathrm{C} \text {, air flow rate } 500 \mathrm{~mL} / \mathrm{min} \\
\text { or } \mathrm{O}_{2} \text { flow rate } 100 \mathrm{~mL} / \mathrm{min} \text { for } 6 \mathrm{~h} \text {; } \\
\text { or } \mathrm{T}=50{ }^{\circ} \mathrm{C}, 2 \mathrm{~g} 30 \% \mathrm{H}_{2} \mathrm{O}_{2} \text { for } 3 \mathrm{~h} ; 5 \\
\text { recycles }\end{array}$ & 117 \\
\hline$\left[\mathrm{C}_{4} \mathrm{mim}\right]_{3} \mathrm{PMo}_{12} \mathrm{O}_{40}$ & {$[\mathrm{Bmim}] \mathrm{Cl}$} & $2000 \mathrm{mg} / \mathrm{m}^{3}$ & - & 100 & $80-180$ & 300 & 100 & - & Air bubbling for $24 \mathrm{~h} ; 6$ recycles & 35 \\
\hline $\begin{array}{l}{[\mathrm{Bmim}] \mathrm{Cl} / \mathrm{CuCl}_{2} / \mathrm{sili}} \\
\text { ca gel }\end{array}$ & - & $1000 \mathrm{mg} / \mathrm{m}^{3}$ & - & 100 & $20-50$ & 66 & 100 & - & $\begin{array}{l}\text { Air flow rate } 100 \mathrm{~mL} / \mathrm{min} \text { for } 5 \mathrm{~h} ; 4 \\
\text { recycles }\end{array}$ & 118 \\
\hline$[\mathrm{Bmim}][\mathrm{Fe}(\mathrm{EDTA})]$ & NHD & $99.9 \%$ & 0.156 & 10 & RT & 420 & - & $\begin{array}{l}4.3 \\
\mathrm{mg} /(\mathrm{mL} \cdot \mathrm{h})\end{array}$ & $\mathrm{RT}, \mathrm{O}_{2}$ flow rate $25 \mathrm{~mL} / \mathrm{min}$ & 119 \\
\hline
\end{tabular}




\section{References}

1. Hayashi, S.; Hamaguchi, H. O. Discovery of a magnetic ionic liquid [Bmim]FeCl 4 . Chem. Lett. 2004, 33 (12), $1590-1591$.

2. Hayashi, S.; Saha, S.; Hamaguchi, H. O. A new class of magnetic fluids: bmim[ $\left.\mathrm{FeCl}_{4}\right]$ and nbmim[FeCl 4$]$ ionic liquids. IEEE T. Magn. 2006, $42(1), 12-14$.

3. Lee, S. H.; Ha, S. H.; Ha, S. S.; Jin, H. B.; You, C. Y.; Koo, Y. M. Magnetic behavior of mixture of magnetic ionic liquid BmimFeCl 4 and water. J. Appl. Phys. 2007, 101 (9), 09J102-09J102-3.

4. Xie, Z. L.; Taubert, A. Thermomorphic behavior of the ionic liquids $\left[\mathrm{C}_{4} \mathrm{mim}\right]\left[\mathrm{FeCl}_{4}\right]$ and $\left[\mathrm{C}_{12} \mathrm{mim}\right]\left[\mathrm{FeCl}_{4}\right]$. Chemphyschem 2011, 12 (2), 364368.

5. Wang, J.; Yao, H.; Nie, Y.; Zhang, X.; Li, J. Synthesis and characterization of the iron-containing magnetic ionic liquids. J. Mol. Liq. 2012, $169,152-155$.

6. Del Sesto, R. E.; McCleskey, T. M.; Burrell, A. K.; Baker, G. A.; Thompson, J. D.; Scott, B. L.; Wilkes, J. S.; Williams, P. Structure and magnetic behavior of transition metal based ionic liquids. Chem. Commun. 2008, 447-449.

7. Pei, Y.; Cao, Y.; Huang, Y.; Song, X.; Wang, H.; Zhao, Y.; Wang, J. Tunable LCST-type phase behavior of [FeCl $]^{-}{ }^{-b a s e d}$ ionic liquids in water. Sci. China Chem. 2016, 59 (5), 587-593.

8. Tian, P.; Song, X. M.; Li, Y.; Duan, J. D.; Liang, Z. D.; Zhang, H. Studies on room temperature ionic liquid $\mathrm{FeCl}_{3}-\mathrm{BPC}_{\mathrm{P}}$ system. Acta Chim. Sinica 2006, 64 (23), 2305-2309.

9. Pierson, S. A.; Nacham, O.; Clark, K. D.; Nan, H.; Mudryk, Y.; Anderson, J. L. Synthesis and characterization of low viscosity hexafluoroacetylacetonate-based hydrophobic magnetic ionic liquids. New J. Chem. 2017, 41 (13), 5498-5505.

10. Wu, Q.; Dong, B.; Han, M.; Xin, H.; Jin, Y. Studies on acidity of chloroaluminate ionic liquids using pyridine as infrared spectroscopic probe. 
Chinese J. Anal. Chem. 2006, 34 (9), 1323-1326.

11. Wu, Q.; Han, M.; Xin, H.; Dong, B.; Jin, Y. Studies on IR spectroscopy and quantum chemical calculation of chloroaluminate ionic liquids acidity. Spectrosc. Spect. Anal. 2008, 28 (2), 282-284.

12. Zhang, X.; Zhang, R.; Liu, H.; Meng, X.; Xu, C.; Liu, Z.; Klusener, P. A. A. Quantitative characterization of Lewis acidity and activity of chloroaluminate ionic liquids. Ind. Eng. Chem. Res. 2016, 55 (46), 11878-11886.

13. Hu, P.; Jiang, W.; Zhong, L.; Zhou, S. F. Determination of the lewis acidity of amide- $\mathrm{AlCl}_{3}$ based ionic liquid analogues by combined in situ IR titration and NMR methods. RSC Adv. 2018, 8 (24), 13248-13252.

14. Sitze, M. S.; Schreiter, E. R.; Patterson, E. V.; Freeman, R. G. Ionic liquids based on $\mathrm{FeCl}_{3}$ and $\mathrm{FeCl}_{2}$. Raman scattering and ab initio calculations. Inorg. Chem. 2001, 40, 2298-2304.

15. Zhang, Q. G.; Yang, J. Z.; Lu, X. M.; Gui, J. S.; Huang, Z. Studies on an ionic liquid based on $\mathrm{FeCl}_{3}$ and its properties. Fluid Phase Equilib. 2004, 226, 207-211.

16. Li, J. G.; Hu, Y. F.; Sun, S. F.; Ling, S.; Zhang, J. Z. Ionic structures of nanobased $\mathrm{FeCl}_{3} /\left[\mathrm{C}_{4} \mathrm{mim}\right] \mathrm{Cl}$ ionic liquids. J. Phys. Chem. B 2012, 116 (22), 6461-6464.

17. Zhou, C.; Yu, X.; Ma, H.; Huang, X.; Zhang, H.; Jin, J. Properties and catalytic activity of magnetic and acidic ionic liquids: Experimental and molecular simulation. Carbohyd. Polym. 2014, 105, 300-307.

18. Wang, T.; Yu, W.; Li, T.; Wang, Y.; Tan, J.; Hu, B.; Nie, L. Synthesis of novel magnetic ionic liquids as high efficiency catalysts for extractioncatalytic oxidative desulfurization in fuel oil. New J. Chem. 2019, 43 (48), 19232-19241.

19. Andevary, H. H.; Akbari, A.; Omidkhah, M. High efficient and selective oxidative desulfurization of diesel fuel using dual-function [Omim] $\mathrm{FeCl}_{4}$ as catalyst/extractant. Fuel Process. Technol. 2019, 185, 8-17. 
20. Yoshida, Y.; Fujii, J.; Muroi, K.; Otsuka, A.; Saito, G.; Takahashi, M.; Yoko, T. Highly conducting ionic liquids based on 1-ethyl-3methylimidazolium cation. Synthetic. Met. 2005, 153 (1-3), 421-424.

21. Wang, K. F.; Zhang, L.; Zhuang, R. R.; Jian, F. F. An iron(III)-containing ionic liquid: Characterization, magnetic property and electrocatalysis. Transit. Metal Chem. 2011, 36 (8), 785-791.

22. Ma, Y.; Wang, R. $\mathrm{H}_{2} \mathrm{~S}$ absorption capacity and regeneration performance of amine Fe-based ionic liquid. Chem. J. Chinese Univ. 2014, 35 (4), $760-765$.

23. Daniel, C. I.; Vaca Chavez, F.; Portugal, C. A. M.; Crespo, J. G.; Sebastiao, P. J. ${ }^{1}$ H NMR relaxation study of a magnetic ionic liquid as a potential contrast agent. J. Phys. Chem. B 2015, 119 (35), 11740-11747.

24. Qian, L.; Hu, X.; Guan, P.; Guo, X., Bi-functional magnetical chiral ionic liquids derived from imidazolium and pyridinium. Adv. Mater. Engin. Appl. 2012, 161, 128-133.

25. Voronchikhina, L. I.; Zhuravlev, O. E.; Verolainen, N. V.; Krotova, N. I. Effect of the cation structure on the thermal stability of ionic liquids, quaternary ammonium tetrachloroferrates(III). Russ. J. Gen. Chem. 2016, 86 (6), 1314-1318.

26. Yu, X.; Yuan, X.; Xia, Z.; Ren, L. Self-assembly of magnetic poly(ionic liquid)s and ionic liquids in aqueous solution. Polym. Chem. 2018, 9 (41), 5116-5122.

27. Dai, X.; Qiang, X.; Li, J.; Yao, T.; Wang, Z.; Song, H. Design and functionalization of magnetic ionic liquids surfactants (MILs) containing alkyltrimethylammonium fragment. J. Mol. Liq. 2019, 277, 170-174.

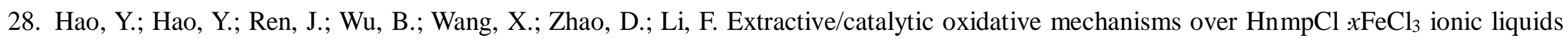
towards the desulfurization of model oils. New J. Chem. 2019, 43 (20), 7725-7732.

29. Zhong, C.; Sasaki, T.; Jimbo-Kobayashi, A.; Fujiwara, E.; Kobayashi, A.; Tada, M.; Iwasawa, Y. Syntheses, structures, and properties of a 
series of metal ion-containing dialkylimidazolium ionic liquids. Bull. Chem. Soc. Jpn. 2007, 80 (12), 2365-2374.

30. Zuerner, P.; Schmidt, H.; Bette, S.; Wagler, J.; Frisch, G. Ionic liquid, glass or crystalline solid? Structures and thermal behaviour of $\left(\mathrm{C}_{4} \mathrm{mim}\right)_{2} \mathrm{CuCl}_{3}$. Dalton Trans. 2016, 45 (8), 3327-3333.

31. Yang, H.; Jiang, B.; Sun, Y.; Hao, L.; Huang, Z.; Zhang, L. Synthesis and oxidative desulfurization of novel lactam-based bronsted-Lewis acidic ionic liquids. Chem. Eng. J. 2016, 306, 131-138.

32. Zhang, L.; Wang, J.; Sun, Y.; Jiang, B.; Yang, H. Deep oxidative desulfurization of fuels by superbase-derived Lewis acidic ionic liquids. Chem. Eng. J. 2017, 328, 445-453.

33. Wang, J.; Zhang, L.; Sun, Y.; Jiang, B.; Chen, Y.; Gao, X.; Yang, H. Deep catalytic oxidative desulfurization of fuels by novel Lewis acidic ionic liquids. Fuel Process. Technol. 2018, 177, 81-88.

34. Zhang, D.; Zhang, W. Q.; Lin, Z. G.; Dong, J.; Zhen, N.; Chi, Y. N.; Hu, C. W. Mono- and Di-Sc-substituted keggin polyoxometalates: Effective lewis acid catalysts for nerve agent simulant hydrolysis and mechanistic insights. Inorg. Chem. 2020, 59 (14), $9756-9764$.

35. Ma, Y.; Liu, X.; Wang, R. Efficient removal of $\mathrm{H}_{2} \mathrm{~S}$ at high temperature using the ionic liquid solutions of $\left[\mathrm{C}_{4} \mathrm{mim}_{3} \mathrm{PMo}_{12} \mathrm{O}_{40}-\mathrm{An}_{0}\right.$ organic polyoxometalate. J. Hazard. Mater. 2017, 331, 109-116.

36. Li, A.; Song, H.; Meng, H.; Lu, Y.; Li, C. Ultrafast desulfurization of diesel oil with ionic liquid based PMoO catalysts and recyclable NaClO oxidant. Chem. Eng. J. 2020, 380, 122453.

37. Akopyan, A.; Eseva, E.; Polikarpova, P.; Kedalo, A.; Vutolkina, A.; Glotov, A. Deep oxidative desulfurization of fuels in the presence of bronsted acidic polyoxometalate-based ionic liquids. Molecules 2020, 25 (3), 536.

38. Hao, L.; Sun, L.; Su, T.; Hao, D.; Liao, W.; Deng, C.; Ren, W.; Zhang, Y.; Lu, H. Polyoxometalate-based ionic liquid catalyst with unprecedented activity and selectivity for oxidative desulfurization of diesel in [Omim]BF 4. Chem. Eng. J. 2019, 358, 419-426. 
39. Li, Y.; Zhang, Y.; Wu, P.; Feng, C.; Xue, G. Catalytic oxidative/extractive desulfurization of model oil using transition metal substituted phosphomolybdates-based ionic liquids. Catalysts 2018, 8 (12), 639.

40. Boglio, C.; Micoine, K.; Remy, P.; Hasenknopf, B.; Thorimbert, S.; Lacote, E.; Malacria, M.; Afonso, C.; Tabet, J. C. Increased Lewis acidity in hafnium-substituted polyoxotungstates. Chem.-Eur. J. 2007, 13 (19), 5426-5432.

41. Ding, Y. X.; Zhu, W. S.; Li, H. M.; Jiang, W.; Zhang, M.; Duan, Y. Q.; Chang, Y. H. Catalytic oxidative desulfurization with a hexatungstate/aqueous $\mathrm{H}_{2} \mathrm{O}_{2}$ /ionic liquid emulsion system. Green Chem. 2011, 13 (5), 1210-1216.

42. Ge, J. H.; Zhou, Y. M.; Yang, Y.; Xue, M. W. Catalytic oxidative desulfurization of gasoline using ionic liquid emulsion system. Ind. Eng. Chem. Res. 2011, 50 (24), 13686-13692.

43. Zhu, W.; Zhu, G.; Li, H.; Chao, Y.; Chang, Y.; Chen, G.; Han, C. Oxidative desulfurization of fuel catalyzed by metal-based surfactant-type ionic liquids. J. Mol. Catal. A: Chem. 2011, 347 (1-2), 8-14.

44. Zhang, B.; Jiang, W.; Fan, X.; Zhang, M.; Wei, Y.; He, J.; Li, C.; Li, H.; Zhu, W. Synthesis of amphiphilic peroxophosphomolybdates for oxidative desulfurization of fuels in ionic liquids. Pet. Sci. 2018, 15 (4), 890-897.

45. Bourlinos, A. B.; Raman, K.; Herrera, R.; Qiang Zhang; Archer, L. A.; Giannelis, E. P. A liquid derivative of 12-tungstophosphoric acid with unusually high conductivity. J. Am. Chem. Soc. 2004, 126, 15358-15359.

46. Rickert, P. G.; Antonio, M. R.; Firestone, M. A.; Kubatko, K. A.; Szreder, T.; Wishart, J. F.; Dietz, M. L. Tetraalkylphosphonium polyoxometalates: Electroactive, "task-specific" ionic liquids. Dalton Trans. 2007, 529-31.

47. Huang, T.; Xie, Z.; Wu, Q.; Yan, W. Temperature-dependent gel-type ionic liquid compounds based on vanadium-substituted polyoxometalates with keggin structure. Dalton Trans. 2016, 45 (9), 3958-63.

48. Santos, F. M.; Magina, S. P.; Nogueira, H. I. S.; Cavaleiro, A. M. V. Synthesis and characterization of metal-substituted tetraalkylphosphonium 
polyoxometalate ionic liquids. New J. Chem. 2016, 40 (2), 945-953.

49. Santos, F. M.; Brandao, P.; Felix, V.; Domingues, M. R. M.; Amaral, J. S.; Amaral, V. S.; Nogueira, H. I. S.; Cavaleiro, A. M. V. Organicinorganic hybrid materials based on iron(III)-polyoxotungstates and 1-butyl-3-methylimidazolium cations. Dalton Trans. 2012, 41 (39), 1214512155.

50. Liu, X.; Li, J.; Wang, R. Desulfurization and regeneration performance of heteropoly compound/ionic liquid solutions at high temperature. Chem. Eng. J. 2017, 316, 171-178.

51. Pratt, H. D.; Rose, A. J.; Staiger, C. L.; Ingersoll, D.; Anderson, T. M. Synthesis and characterization of ionic liquids containing copper, manganese, or zinc coordination cations. Dalton Trans. 2011, 40 (43), 11396-11401.

52. Anderson, T. M.; Ingersoll, D.; Rose, A. J.; Staiger, C. L.; Leonard, J. C. Synthesis of an ionic liquid with an iron coordination cation. Dalton Trans. 2010, 39 (37), 8609-8612.

53. Zhang, P.; Gong, Y.; Lv, Y.; Guo, Y.; Wang, Y.; Wang, C.; Li, H. Ionic liquids with metal chelate anions. Chem. Commun. 2012, 48 (17), 23342336.

54. Teramoto, K.; Nishide, T.; Okumura, S.; Takao, K.; Ikeda, Y. Studies on metal complexes as active materials in redox-flow battery using ionic liquids as electrolyte: Electrochemical properties of $\left[\mathrm{Fe}(\mathrm{L})_{x}\right]\left[\mathrm{Tf}_{2} \mathrm{~N}\right]_{2}$ (L: Multidentate ligands, $x=2$ or 3) in 1-butyl-3-methylimidazolium bis(trifluoromethylsulfonyl)imide, [BmI][Tf 2 N]. Electrochem. 2014, 82 (7), 566-572.

55. Kostopoulos, N.; Achaibou, C.; Noel, J. M.; Kanoufi, F.; Robert, M.; Fave, C.; Anxolabehere-Mallart, E. Electrocatalytic $\mathrm{O}_{2}$ activation by Fe tetrakis(pentafluorophenyl)porphyrin in acidic organic media. Evidence of high-valent fe oxo species. Inorg. Chem. 2020, 59 (16), 11577-11583. 56. Martin, D. J.; Wise, C. F.; Pegis, M. L.; Mayer, J. M. Developing scaling relationships for molecular electrocatalysis through studies of Feporphyrin-catalyzed $\mathrm{O}_{2}$ reduction. Acc. Chem. Res. 2020, 53 (5), 1056-1065. 
57. Ding, F.; Zheng, J.; Chen, Y.; Chen, K.; Cui, G.; Li, H.; Wang, C. Highly efficient and reversible $\mathrm{SO}_{2}$ capture by surfactant-derived dual functionalized ionic liquids with metal chelate cations. Ind. Eng. Chem. Res. 2014, 53 (48), 18568-18574.

58. Yu, G. R.; Zhao, J. J.; Song, D. D.; Asumana, C.; Zhang, X. Y.; Chen, X. C. Deep oxidative desulfurization of diesel fuels by acidic ionic liquids. Ind. Eng. Chem. Res. 2011, 50 (20), 11690-11697.

59. Chen, X.; Song, D.; Asumana, C.; Yu, G. Deep oxidative desulfurization of diesel fuels by lewis acidic ionic liquids based on 1-n-butyl-3methylimidazolium metal chloride. J. Mol. Catal. A: Chem. 2012, 359, 8-13.

60. Nejad, N. F.; Shams, E.; Amini, M. K. Ionic liquid-based extraction of sulfur compounds from gasoline as a complementary process to oxidative desulfurization. Pet. Sci. Technol. 2014, 32 (13), 1537-1544.

61. Dharaskar, S. A.; Wasewar, K. L.; Varma, M. N.; Shende, D. Z. $\mathrm{FeCl}_{3}$ based imidazolium ionic liquids as novel solvents for extractiveoxidative desulfurization of liquid fuels. J. Solution Chem. 2015, 44 (3-4), 652-668.

62. Zhang, Y.; Xu, H.; Jia, M.; Liu, Z.; Qu, D. Optimization of deep oxidative desulfurization process using ionic liquid and potassium monopersulfate. J. Chem. 2018, 2018, 1-6.

63. Ban, L. L.; Liu, P.; Ma, C. H.; Dai, B. Deep extractive desulfurization of diesel fuels by $\mathrm{FeCl}_{3} /$ ionic liquids. Chin. Chem. Lett. 2013, 24 (8), $755-758$.

64. Gao, L.; Dai, Z.; Li, G. Oxidative desulfurization of fuels catalyzed by schiff-base cobalt complex in Fe-based ionic liquid. Chem. Ind. Eng. Pro. (China) 2014, 33 (12), 3393-3398.

65. Guo, Y. F.; Gao, C. Y.; Yang, K. G.; Wang, Z. J.; Duan, Y. H.; Feng, S. X.; Xu, H. Mild and deep oxidative extraction desulfurization using dual-function imidazolium peroxydisulfate ionic liquid. Energy Fuels 2019, 33 (11), 10728-10733.

66. Nie, Y.; Gong, X.; Gao, H.; Zhang, X.; Zhang, S. Simultaneous desulfurization and denitrogen of liquid fuels using two functionalized group 
ionic liquids. Sci. China Chem. 2014, 57 (12), 1766-1773.

67. Chen, X.; Guan, Y.; Abdeltawab, A. A.; Al-Deyab, S. S.; Yuan, X.; Wang, C.; Yu, G. Using functional acidic ionic liquids as both extractant and catalyst in oxidative desulfurization of diesel fuel: An investigation of real feedstock. Fuel 2015, 146, 6-12.

68. Jiang, Y.; Zhu, W.; Li, H.; Yin, S.; Liu, H.; Xie, Q. Oxidative desulfurization of fuels catalyzed by Fenton-like ionic liquids at room temperature. Chemsuschem 2011, 4 (3), 399-403.

69. Zhu, W.; Zhang, J.; Li, H.; Chao, Y.; Jiang, W.; Yin, S.; Liu, H. Fenton-like ionic liquids $/ \mathrm{H}_{2} \mathrm{O}_{2}$ system: One-pot extraction combined with oxidation desulfurization of fuel. RSC Adv. 2012, 2 (2), 658-664.

70. Jiang, W.; Zhu, W. S.; Li, H. M.; Xiong, J.; Xun, S. H.; Zhao, Z.; Wang, Q. Deep oxidative desulfurization of fuels catalyzed by magnetic Fenton-like hybrid catalysts in ionic liquids. RSC Adv. 2013, 3 (7), 2355-2361.

71. Wang, S.; Li, P.; Hao, L.; Deng, C.; Ren, W.; Lu, H. Oxidative desulfurization of model diesel using a Fenton-like catalyst in the ionic liquid [Dmim]BF 4 . Chem. Eng. Technol. 2017, 40 (3), 555-560.

72. Li, F. T.; Kou, C. G.; Sun, Z. M.; Hao, Y. J.; Liu, R. H.; Zhao, D. S. Deep extractive and oxidative desulfurization of dibenzothiophene with $\mathrm{C}_{5} \mathrm{H}_{9} \mathrm{NO} \cdot \mathrm{SnCl}_{2}$ coordinated ionic liquid. J. Hazard. Mater. 2012, 205, 164-170.

73. Dong, Y. X.; Nie, Y.; Zhou, Q. Highly efficient oxidative desulfurization of fuels by Lewis acidic ionic liquids based on iron chloride. Chem. Eng. Technol. 2013, 36 (3), 435-442.

74. Nie, Y.; Dong, Y. X.; Bai, L.; Dong, H. F.; Zhang, X. P. Fast oxidative desulfurization of fuel oil using dialkylpyridinium tetrachloroferrates ionic liquids. Fuel 2013, 103, 997-1002.

75. Zhu, W.; Wu, P.; Yang, L.; Chang, Y.; Chao, Y.; Li, H.; Jiang, Y.; Jiang, W.; Xun, S. Pyridinium-based temperature-responsive magnetic ionic liquid for oxidative desulfurization of fuels. Chem. Eng. J. 2013, 229, 250-256. 
76. Jiang, W.; Zhu, W. S.; Chang, Y. H.; Chao, Y. H.; Yin, S.; Liu, H.; Zhu, F. X.; Li, H. M. Ionic liquid extraction and catalytic oxidative desulfurization of fuels using dialkylpiperidinium tetrachloroferrates catalysts. Chem. Eng. J. 2014, 250, 48-54.

77. Jiang, W.; Zhu, W. S.; Li, H. M.; Chao, Y. H.; Xun, S. H.; Liu, H.; Zhao, Z. Mechanism and optimization for oxidative desulfurization of fuels catalyzed by fenton-like catalysts in hydrophobic ionic liquid. J. Mol. Catal. A: Chem. 2014, 382, 8-14.

78. Chen, X.; Guo, H.; Abdeltawab, A. A.; Guan, Y.; Al-Deyab, S. S.; Yu, G.; Yu, L. Brönsted-Lewis acidic ionic liquids and application in oxidative desulfurization of diesel fuel. Energy Fuels 2015, 29 (5), 2998-3003.

79. Singh, S.; Srivastava, V. C.; Gautam, S. Oxidative-extractive desulfurization of liquid fuel by dimethyl sulfoxide and $\mathrm{ZnCl}_{2}$ based ionic liquid. Int. J. Chem. React. Eng. 2016, 14 (1), 539-545.

80. Zhu, W. S.; Ding, Y. X.; Li, H. M.; Qin, J.; Chao, Y. H.; Xiong, J.; Xu, Y. H.; Liu, H. Application of a self-emulsifiable task-specific ionic liquid in oxidative desulfurization of fuels. RSC Adv. 2013, 3 (12), 3893-3898.

81. Huang, W. L.; Zhu, W. S.; Li, H. M.; Shi, H.; Zhu, G. P.; Liu, H.; Chen, G. Y. Heteropolyanion-based ionic liquid for deep desulfurization of fuels in ionic liquids. Ind. Eng. Chem. Res. 2010, 49 (19), 8998-9003.

82. Zhu, W. S.; Huang, W. L.; Li, H. M.; Zhang, M.; Jiang, W.; Chen, G. Y.; Han, C. R. Polyoxometalate-based ionic liquids as catalysts for deep desulfurization of fuels. Fuel Process. Technol. 2011, 92 (10), 1842-1848.

83. Rafiee, E.; Mirnezami, F. Keggin-structured polyoxometalate-based ionic liquid salts: Thermoregulated catalysts for rapid oxidation of sulfurbased compounds using $\mathrm{H}_{2} \mathrm{O}_{2}$ and extractive oxidation desulfurization of sulfur-containing model oil. J. Mol. Liq. 2014, 199, $156-161$.

84. Yu, F. L.; Tang, H. B.; Liu, C. Y.; Xie, C. X.; Yu, S. T. Oxidative desulfurization of model diesel with $\mathrm{O}_{2}$ catalyzed by oxidativethermoregulated bifunctional ionic liquids. Acta Chim. Sinica 2014, 72 (11), 1152-1156.

85. Yu, F. L.; Wang, Y. Y.; Liu, C. Y.; Xie, C. X.; Yu, S. T. Oxidative desulfurization of fuels catalyzed by ammonium oxidative-thermoregulated 
bifunctional ionic liquids. Chem. Eng. J. 2014, 255, 372-376.

86. Chen, J.; Chen, C.; Zhang, R.; Guo, L.; Hua, L.; Chen, A.; Xiu, Y.; Liu, X.; Hou, Z. Deep oxidative desulfurization catalyzed by an ionic liquid-type peroxotungsten catalyst. RSC Adv. 2015, 5 (33), 25904-25910.

87. Juliao, D.; Valenca, R.; Ribeiro, J. C.; de Castro, B.; Balula, S. S. Efficient eco-sustainable ionic liquid-polyoxometalate desulfurization processes for model and real diesel. Appl. Catal., A. 2017, 537, 93-99.

88. Liu, H.; Xu, H.; Hua, M.; Chen, L.; Wei, Y.; Wang, C.; Wu, P.; Zhu, F.; Chu, X.; Li, H.; Zhu, W. Extraction combined catalytic oxidation desulfurization of petcoke in ionic liquid under mild conditions. Fuel 2020, 260, 116200.

89. Xu, Y.; Ma, W. W.; Dolo, A.; Zhang, H. An amphiphilic catalyst based on sandwich-type polyoxometalate for deep desulfurization of fuels in ionic liquid. RSC Adv. 2016, 6 (71), 66841-66846.

90. Yu, F.; Xie, P.; Zhu, G.; Yuan, B.; Xie, C.; Yu, S. Oxidative desulfurization of gasoline catalyzed by organic-inorganic heteropoly acid ionic liquids under ultrasound. Chem. J. Chinese Univ. 2016, 37 (12), 2184-2190.

91. Xu, J. H.; Zhao, S.; Ji, Y. C.; Song, Y. F. Deep desulfurization by amphiphilic Lanthanide-containing polyoxometalates in ionic-liquid emulsion systems under mild conditions. Chem.-Eur. J. 2013, 19 (2), 708-714.

92. Ge, J.; Zhou, Y.; Yang, Y.; Xue, M. The deep oxidative desulfurization of fuels catalyzed by surfactant-type octamolybdate in acidic ionic liquids. Pet. Sci. Technol. 2014, 32 (1), 116-123.

93. Lu, H. Y.; Deng, C. L.; Ren, W. Z.; Yang, X. Oxidative desulfurization of model diesel using $\left[\left(\mathrm{C}_{4} \mathrm{H}_{9}\right)_{4} \mathrm{~N}_{6} \mathrm{Mo}_{7} \mathrm{O}_{24}\right.$ as a catalyst in ionic liquids. Fuel Process. Technol. 2014, 119, 87-91.

94. Shao, B. B.; Shi, L.; Meng, X. Deep desulfurization of 4,6-dimethyldienzothiophene by an ionic liquids extraction coupled with catalytic oxidation with a molybdic compound. Ind. Eng. Chem. Res. 2014, 53 (16), 6655-6663. 
95. Fareghi-Alamdari, R.; Zekri, N.; Moghadam, A. J.; Farsani, M. R. Green oxidation of sulfides to sulfoxides and sulfones with $\mathrm{H}_{2} \mathrm{O}_{2}$ catalyzed by ionic liquid compounds based on keplerate polyoxometalates. Catal. Commun. 2017, 98, 71-75.

96. Akopyan, A. V.; Eseva, E. A.; Polikarpova, P. D.; Baigil'diev, T. M.; Rodin, I. A.; Anishnov, A. V. Catalytic activity of polyfunctional ionic liquids in oxidation of model sulfur organic compounds. Russ. J. Appl. Chem. 2019, 92 (4), 569-575.

97. Jiang, W.; Jia, H.; Zheng, Z.; Zhu, L.; Dong, L.; Liu, W.; Zhu, W.; Li, H. Catalytic oxidative desulfurization of fuels in acidic deep eutectic solvents with $\left[\left(\mathrm{C}_{6} \mathrm{H}_{13}\right)_{3} \mathrm{P}\left(\mathrm{C}_{14} \mathrm{H}_{29}\right)\right]_{3} \mathrm{PMo}_{12} \mathrm{O}_{40}$ as a catalyst. Pet. Sci. 2018, 15 (4), 841-848.

98. Ge, J. H.; Zhou, Y. M.; Yang, Y.; Xue, M. W. Catalytic oxidative desulfurization of gasoline using vanadium (V)-substituted polyoxometalate $/ \mathrm{H}_{2} \mathrm{O}_{2}$ /ionic liquid emulsion system. China Pet. Process. Petrochem. Technol. 2012, 14 (1), 25-31.

99. Li, J. L.; Hu, B.; Hu, C. Q. Deep desulfurization of fuels by heteropolyanion-based ionic liquid. Bull. Korean Chem. Soc. 2013, 34 (1), 225230.

100.Lu, H. Y.; Ren, W. Z.; Wang, H. Y.; Wang, Y.; Chen, W.; Suo, Z. H. Deep desulfurization of diesel by ionic liquid extraction coupled with catalytic oxidation using an anderson-type catalyst $\left[\left(\mathrm{C}_{4} \mathrm{H}_{9}\right)_{4} \mathrm{~N}\right]_{4} \mathrm{NiMo}_{6} \mathrm{O}_{24} \mathrm{H}_{6}$. Appl. Catal., A. 2013, 453, 376-382.

101.Sun, L.; Su, T.; Li, P.; Xu, J.; Chen, N.; Liao, W.; Deng, C.; Ren, W.; Lu, H. Extraction coupled with aerobic oxidative desulfurization of model diesel using a B-type Anderson polyoxometalate catalyst in ionic liquids. Catal. Lett. 2019, 149 (7), 1888-1893.

102.Zhang, B.; Li, S.; Yue, S.; Cokoja, M.; Zhou, M. D.; Zang, S. L.; Kuehn, F. E. Imidazolium perrhenate ionic liquids as efficient catalysts for the selective oxidation of sulfides to sulfones. J. Organomet. Chem. 2013, 744, 108-112.

103.Zhao, R.; Wang, J.; Zhang, D.; Sun, Y.; Han, B.; Tang, N.; Wang, N.; Li, K. Biomimetic oxidative desulfurization of fuel oil in ionic liquids catalyzed by $\mathrm{Fe}(\mathrm{III})$ porphyrins. Appl. Catal., A. 2017, 532, 26-31.

104.Zhao, R.; Wang, J.; Zhang, D.; Sun, Y.; Han, B.; Tang, N.; Zhao, J.; Li, K. Deep catalytic oxidative desulfurization of model fuel based on 
modified iron porphyrins in ionic liquids: Anionic ligand effect. ACS Sustainable Chem. Eng. 2017, 5 (3), $2050-2055$.

105.He, Y.; Yu, J.; Chen, L. Wet oxidation desulfurization of hydrogen sulfide with application of Fe-based ionic liquid. J. Chem. Ind. Eng. (China) 2010, 61 (4), 963-968.

106.Yao, R.; Li, P.; Sun, L.; He, Y.; Chen, L.; Yu, Y.; Mu, R.; Yu, J. Physicochemical properties of iron-based chloride imidazole ionic liquid and wet desulfurization mechanism of hydrogen sulfide. J. China Coal Soc. 2011, 36 (1), 135-139.

107.Wang, J.; Zhu, L. Catalytic oxidation of hydrogen sulfide via [bmim] $\mathrm{FeCl}_{4}$ ionic liquid. Nat. Gas Chem. Ind. 2012,37 (06), $29-32$.

108.Ge, X.; Li, Y.; Hu, J.; Zhang, T.; Yu, J. Pilot test design of oxidation desulfurization with application of Fe-based ionic liquids. J. Chem. Ind. Eng. (China) 2013, 64 (S1), 170-174.

109.Wang, J.; Zhang, W. Oxidative absorption of hydrogen sulfide by iron-containing ionic liquids. Energy Fuels 2014, 28 (9), $5930-5935$.

110.Li, W.; Wu, J.; Wang, N.; Chen, L.; Qiu, K.; He, B. Absorption of high concentration $\mathrm{H}_{2} \mathrm{~S}$ by ionic liquid [Bmim]FeCl 4 . Nat. Gas Chem. Ind. 2018, 43 (03), 24-28.

111.Wang, J.; Ding, R. Effect of water content on properties of homogeneous [bmim] Fe(III) $\mathrm{Cl}_{4}-\mathrm{H}_{2} \mathrm{O}$ mixtures and their application in oxidative absorption of $\mathrm{H}_{2} \mathrm{~S}$. Inorganics 2018, 6 (1), 11.

112. Wang, J.; Liu, Z.; Tang, L. Catalytic oxidation of $\mathrm{H}_{2} \mathrm{~S}$ by acidic iron(III)-containing ionic liquids. Nat. Gas Chem. Ind. 2018, 43 (04), $41-47$.

113.Guo, Z.; Zhang, T.; Liu, T.; Du, J.; Jia, B.; Gao, S.; Yu, J. Nonaqueous system of iron-based ionic liquid and DMF for the oxidation of hydrogen sulfide and regeneration by electrolysis. Environ. Sci. Technol. 2015, 49 (9), 5697-5703.

114.Hu, J.; Gao, L.; Liu, W.; Zhao, Y.; Gao, S.; Pan, X.; Guo, Z. Effect of components of ionic liquids on oxidation desulfurization performance of hydrogen sulfur. J. Chem. Ind. Eng. (China) 2016, 67 (S1), 347-352.

115.Li, M.; Guan, J.; Han, J.; Liang, W.; Wang, K.; Duan, E.; Guo, B. Absorption and oxidation of $\mathrm{H}_{2} \mathrm{~S}$ in triethylamine hydrochloride · ferric 
chloride ionic liquids. J. Mol. Liq. 2015, 209, 58-61.

116. Wang, J.; Yu, X.; Zhan, M.; Xu, B.; Zhu, L.; Wang, Y. Oxidation absorption of $\mathrm{H}_{2} \mathrm{~S}$ by [Bmim]OH and [ $\left.\mathrm{A}_{336}\right]\left[\mathrm{FeCl}_{4}\right]$ mixed ionic liquids. Nat. Gas Ind. 2018, 38 (07), 100-107.

117.Liu, X.; Wang, R. $\mathrm{H}_{2}$ S removal by peroxo heteropoly compound/ionic liquid solution. Fuel Process. Technol. 2017,160 , 78-85.

118. Ma, Y.; Mao, J.; Xiao, C.; Li, Y.; Zang, L. Immobilization of functionalized ionic liquid on sol-gel derived silica for efficient removal of $\mathrm{H}_{2} \mathrm{~S}$.

China Pet. Process. Petrochem. Technol. 2019, 21 (1), 62-70.

119.Wang, J.; Wan, Y.; Zhang, Y. An absorbing agent for desulfurization and decarbonization: CHN Patent 109432990 A[P], $2019-03-08$. 